ELECTROPHYSIOLOGICAL CORRELATES OF ORIENTING RETRIEVAL TO

REMOTE AND RECENT MEMORIES:

EFFECTS OF AN EPISODIC SPECIFICITY INDUCTION

A Dissertation

presented to

the Faculty of the Graduate School

at the University of Missouri

In Partial Fulfillment

of the Requirements for the Degree

Doctor of Philosophy

by

EMILY K. LEIKER

Dr. Jeffrey D. Johnson, Dissertation Supervisor

MAY 2017 
The undersigned, appointed by the Dean of the Graduate School, have examined the dissertation entitled:

\section{ELECTROPHYSIOLOGICAL CORRELATES OF ORIENTING RETRIEVAL TO}

REMOTE AND RECENT MEMORIES:

\section{EFFECTS OF AN EPISODIC SPECIFICITY INDUCTION}

presented by Emily K. Leiker,

a candidate for the degree of doctor of philosophy,

and hereby certify that, in their opinion, it is worthy of acceptance.

Dr. Jeffrey D. Johnson

Dr. Bruce D. Bartholow

Dr. Roxana Botezatu

Dr. Steven A. Hackley 
To my favorite people: Stephen and Huxley.

Thank you for your endless love and support, and for encouraging me to stop and smell the flowers along the way. 


\section{ACKNOWLEDGEMENTS}

Thank you to my advisor, Jeff Johnson, for your enduring support and guidance throughout my dissertation and over the course of my graduate career. I am truly grateful for your advice and encouragement, and for providing a listening ear as I navigated the hurdles and hiccups that arose along the way. Your patience and compassion, and your willingness to sit down and talk things through until I understood them, have been invaluable to my growth and development in science and research.

I would also like to thank the members of my dissertation committee, Drs. Bruce Bartholow, Roxana Botezatu, and Steve Hackley, for their instructive feedback and insight as to additional interpretations and follow-up questions for the present set of experiments. The supportive and constructive nature of their comments and suggestions ensured that this challenging process was both informative and rewarding.

Thanks to everyone in the Memory and Neuroimaging Lab, especially those who helped me with data collection over the years. Thank you to my labmate Mason, for your support and camaraderie, and for helping me take a break once in awhile to appreciate whatever hilarity was going on in the world. All of you made me look forward to going to work each day.

Thank you to my friends and family, without whose support my journey through graduate school would have been far more arduous. I am so grateful to have had a cohort of intelligent, kind, and funny individuals to turn to for support, vent sessions, and fun. I will always cherish the laughter and friendship we shared as we progressed through our degrees. I can't wait to see what each of you does next. 
And finally, thank you to Stephen - the love of my life and my partner in crime. You have made the last five years the best years of my life so far. Thanks for being silly with me and helping me to find the joy through all of life's twists and turns. I couldn't imagine a better person to share this crazy adventure of life with, and I can't wait to see where it takes us. Thank you for always being there when I needed you, even when I was too stubborn to admit it. It's been a wild ride, and I'm so glad I got to share it with you. 


\section{TABLE OF CONTENTS}

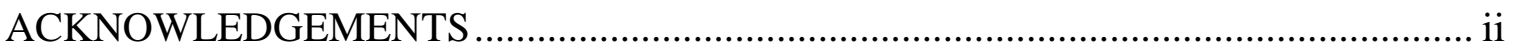

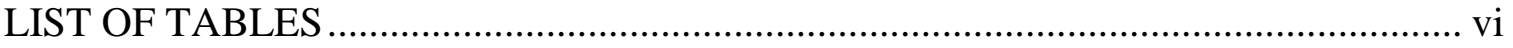

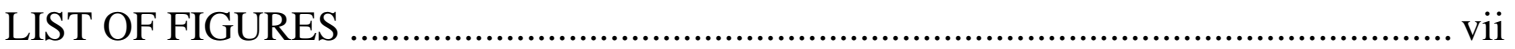

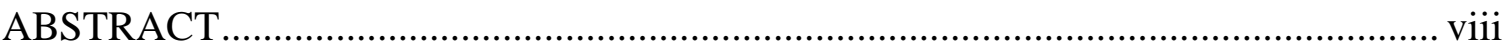

Chapter

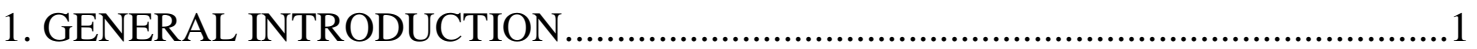

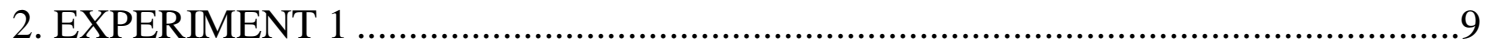

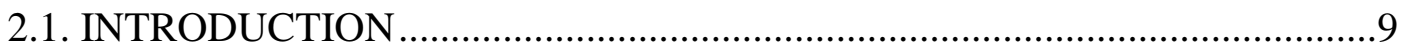

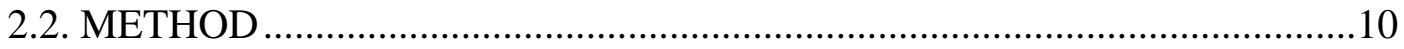

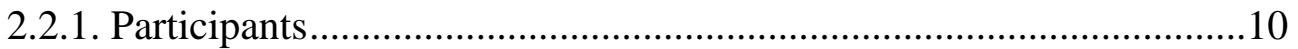

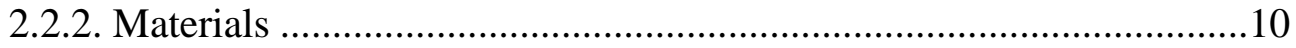

2.2.3. Design and Procedure ..............................................................11

2.2.4. EEG Acquisition, Processing, and Analysis ..................................13

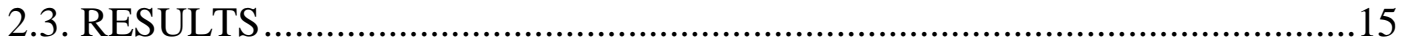

2.3.1. Behavioral Results ...................................................................15

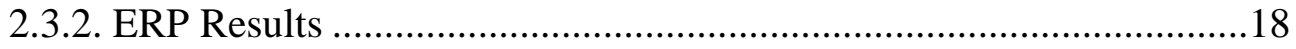

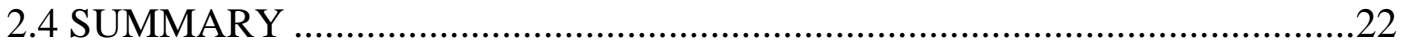

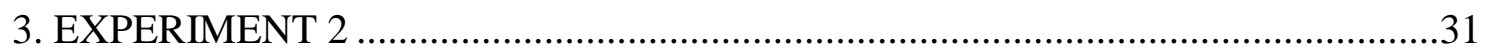

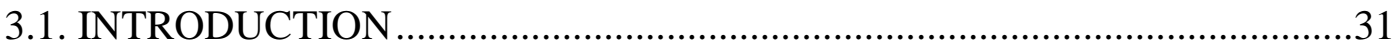

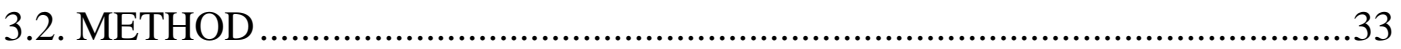

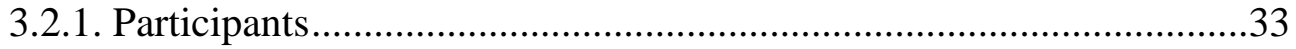


3.2.2. Materials

3.2.3. Design and Procedure .34

3.2.4. EEG Acquisition, Processing, and Analysis .36

3.3. RESULTS .37

3.3.1. Behavioral Results .37

3.3.2. ERP Results .39

3.4. SUMMARY .43

4. GENERAL DISCUSSION .51 REFERENCES .58

VITA .72 


\section{LIST OF TABLES}

Table

Page

1. Mean proportions of correct responses and corresponding RTs during the targeted

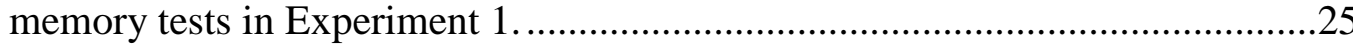

2. Mean proportions of correct responses and corresponding RTs during the final test

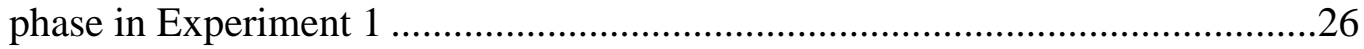

3. Mean proportions of correct responses and corresponding RTs during the targeted memory tests in Experiment 2 .....................................................................45 


\section{LIST OF FIGURES}

Figure

1. Mean ERPs during Remote-4x and Recent-1x orienting in Experiment 1

2. Difference waveforms for the Recent $-1 x$ minus Remote- $4 x$ contrast in

Experiment 1

3. Topographic maps for the Recent-1x minus Remote-4x contrast in

Experiment 1

4. Mean ERPs during Remote-4x and Remote-1x orienting in Experiment 1

5. Mean ERPs during Remote-General and Recent-Specific orienting in Experiment 2

6. Topographic maps for the Recent-Specific minus Remote-General contrast in Experiment 2

7. Mean ERPs during Remote-General and Remote-Specific orienting in Experiment 2

8. Mean ERPs during all four orienting conditions in Experiment 2 ......

9. Topographic maps for the Recent-General minus Remote-General contrast in Experiment 2 


\title{
ELECTROPHYSIOLOGICAL CORRELATES OF ORIENTING RETRIEVAL TO \\ REMOTE AND RECENT MEMORIES: \\ EFFECTS OF AN EPISODIC SPECIFICITY INDUCTION
}

\author{
Emily K. Leiker \\ Dr. Jeffrey D. Johnson, Dissertation Supervisor
}

\begin{abstract}
The present set of experiments employed event-related brain potentials (ERPs) to investigate differences in how individuals orient retrieval to memories from different times. Experiment 1 investigated whether ERP effects of orienting to remote and recent memories were distinct from effects of retrieval difficulty by manipulating the repetition of studied items across two separate encoding sessions one week apart. Participants then completed a series of targeted retrieval blocks that distinctly probed memory for items from one of each condition. Contrasting the ERPs to correctly rejected new items from the difficulty-matched orienting conditions revealed a positive-going waveform for recent versus remote orienting that differed from the effect of difficulty in both timing and direction. These results replicate prior findings on orienting retrieval to time and indicate that such effects are dissociable from retrieval difficulty. Experiment 2 examined the functional nature of the time-related orienting effects by manipulating the degree of detailed processing engaged prior to targeted retrieval, through a novel computerized procedure for inducing general and specific episodic processing. Although the orienting effects in Experiment 2 were notably weaker than prior findings, the induction reliably modulated behavioral performance and the ERPs for orienting to recent memories. The findings are discussed in the context of further investigating the effectiveness of
\end{abstract}


induction procedures on the processes supporting episodic retrieval, which could have important implications for addressing memory impairment associated with healthy aging and clinical disorders. 


\section{Chapter 1}

\section{GENERAL INTRODUCTION}

A long-standing observation within episodic memory research is that as a memory gets older, it becomes less accessible or even forgotten (Schmolck, Buffalo, \& Squire, 2000; Squire, 1989; for reviews, see Roediger, Weinstein, \& Agarwal, 2010; Wixted, 2004). To date, much of the research attempting to account for this relationship has focused on either the encoding processes that give rise to more durable memory traces that are resistant to the effects of time, or the mechanisms of consolidation, decay, and interference that change memory content and quality over time (Dewar, Cowan, \& Della Sala, 2007; Dudai, 2004; Frankland \& Bontempi, 2005; McGaugh, 2000; Wixted, 2005). Although the work in these areas has been invaluable in advancing our understanding of memory encoding and storage, there has been comparatively less research on retrieval processes that support recovery of memories from different times, and how these processes might be selectively employed to guide memory search. Thus far, most of the research on the subject has focused on characterizing age-related differences in retrieval processing for autobiographical memories in healthy elderly and young adults (e.g., Burke \& Light, 1981; Levine, Svoboda, Hay, Winocur, \& Moscovitch, 2002; Piolino, Desgranges, Benali, \& Eustache, 2002; see Luo \& Craik, 2008, for a review). These studies typically find impaired memory for episodic content as retention interval (or the age of the memory) increases, in line with theories of forgetting (Davis, Small, Stern, 
Mayeux, Feldstein, \& Keller, 2003; Friedman, de Chastelaine, Nessler, \& Malcolm, 2010; for reviews, see Balota, Dolan, \& Duchek, 2000; Friedman, 2000). However, there is increasing evidence that differences in memory performance associated with retention interval and healthy aging may depend to a certain extent on differences in strategic processing employed in the service of retrieval (Morcom \& Rugg, 2004; Rudoy, Weintraub, \& Paller, 2009; for reviews, see Buckner, 2003, 2004; Morcom, 2016; Wilckens, Erickson, \& Wheeler, 2012). Thus, the experiments described herein aim to characterize the functional nature of the strategies engaged during memory retrieval, and to dissociate these strategies from other processes that differ according to memory age.

Distinguishing between the strategic processes that support memory retrieval and those that directly signify retrieval dates back to the concept of "retrieval mode" proposed by Tulving (1983). According to this idea, retrieval mode reflects the processing of incoming stimuli as retrieval cues for the purpose of accessing a target memory, as opposed to simply processing the incoming stimuli for encoding (Rugg \& Wilding, 2000; Tulving, 1983). Whereas retrieval mode represents a cognitive state engaged at a general level of the memory search, a more specific form of strategic retrieval processing was later proposed that allows retrieval cues to more effectively access memories comprised of particular kinds of information, or which were encoded under particular task demands (Johnson, Kounios, \& Nolde, 1997; Morris, Bransford, \& Franks, 1977; Rugg \& Wilding, 2000; Tulving \& Thomson, 1973). This cognitive state of retrieval orientation, which is of central interest in the present experiments, is likely to occur when an individual has prior knowledge or expectation about the nature of the targeted memory (e.g., regarding characteristics or qualities of the episodic content or the 
manner of encoding). Orienting thereby allows the retrieval search to be constrained to target memory traces with the greatest relevance via strategic processing of the retrieval cue (Herron \& Rugg, 2003a; Jacoby, Shimizu, Daniels, \& Rhodes, 2005; Robb \& Rugg, 2002; Shimizu \& Jacoby, 2005). In other words, adopting a particular retrieval orientation is as a form of proactive selection that allows individuals to process retrieval cues in a highly specific manner, increasing the overlap between a cue and the target memory trace to improve likelihood of retrieval success (Herron \& Wilding, 2004; Hornberger, Morcom, \& Rugg, 2004; Rugg \& Wilding, 2000; also see Jacoby, Kelley, \& McElree, 1999; Rugg, Johnson, Park \& Uncapher, 2008; Tulving \& Osler, 1968).

Most of the evidence for the adoption of different retrieval orientations comes from studies employing event-related potentials (ERPs) to monitor brain activity during attempts to retrieve items encoded in different forms (e.g., words vs. pictures; see Herron \& Rugg, 2003a; Johnson \& Rugg, 2006a, 2006b; Robb \& Rugg, 2002; Rugg, Allan, \& Birch, 2000). There are three important features to these studies, which we also employ here. First, the different types of memories are distinctly targeted in separate retrieval blocks to promote the sustained engagement of distinct cognitive sets (Robb \& Rugg, 2002; Wilding \& Nobre, 2001; but see Herron, Evans, \& Wilding, 2016, for an updated approach). Second, the retrieval cues are presented in a single physical format across all targeted memory blocks to avoid confounding retrieval orientation effects with inherent differences in the retrieval cues for the different types of memories (Herron \& Rugg, 2003a; Johnson et al., 1997; Robb \& Rugg, 2002; Rugg et al., 2000; Wilding, 1999; Wilding \& Nobre, 2001). For example, object names can be employed as retrieval cues to target items previously studied in word or picture form (e.g., Herron \& Rugg, 2003a; 
Robb \& Rugg, 2002). Finally, these studies typically focus the analysis on ERPs elicited by only new (non-studied) items on the retrieval test (Herron \& Rugg, 2003a; Robb \& Rugg, 2002; Rugg et al., 2000). Restricting the ERP analysis to correctly rejected new items in this manner avoids potential confounds due to differences in rate of retrieval success or post-retrieval processing across the targeted memory types (Rugg \& Wilding, 2000). By adopting the features outlined above, Robb and Rugg (2002) identified a retrieval orientation effect beginning by around $500 \mathrm{~ms}$ post-stimulus onset, with correctly rejected new items exhibiting a relatively positive waveform when the targets were words compared to pictures. A number of other studies have since identified similar retrieval orientation effects using this approach (Dzulkifli, Sharpe, \& Wilding, 2004; Dzukifli \& Wilding, 2005; Herron \& Wilding, 2005; Johnson \& Rugg, 2006a, 2006b; Rosburg, Johansson \& Mecklinger, 2013; Rosburg, Johansson, Sprondel, \& Mecklinger, 2014).

Whereas the aforementioned approach has been used extensively to study retrieval orientation for memories associated with different modalities (e.g., Johnson \& Rugg, 2006a, 2006b; Robb \& Rugg, 2002) and different encoding tasks (e.g., Dzulkifli et al., 2004; Wilding, 1999), it has recently been adapted to investigate orientation effects related to memories of different ages. In an initial study, Roberts, Tsivilis, and Mayes (2014) investigated retrieval orientation in the context of pictures that were encoded either 48 hours or 40 minutes earlier. A blocked test procedure was employed in which some blocks tested discrimination of target items from only one encoding period versus new items (pure blocks), and others targeted items more generally from both encoding periods (mixed blocks). Whereas Roberts et al. identified widespread topographic 
differences between the correctly rejected new-item ERPs from pure versus mixed blocks, a similar comparison for the two types of pure blocks (48 hours vs. 40 minutes) did not yield any systematic differences. Although the pure versus mixed block effect suggests participants may use memory age to orient retrieval in a general manner, the absence of an effect in the 48-hour versus 40-minute pure block comparison leaves the issue of whether participants can differentially orient retrieval processing to items from a specific encoding time unresolved. Furthermore, because participants only needed to discriminate between new items and old items from one specific encoding period within the pure blocks, this leaves open the possibility that participants may have employed a simple recognition-based strategy to complete the task, rather than adopting a retrieval orientation to memory age (Johnson \& McGhee, 2015; also see Johnson \& Rugg, 2006b).

The above caveat can be avoided by using an exclusion task procedure (Jacoby, 1991, 1998; Jacoby, Toth, \& Yonelinas, 1993) for the retrieval test. In this procedure, participants are instructed to respond positively to studied items of a particular context, while rejecting all other studied items (nontargets) and non-studied (new) items. A recognition-based strategy would be insufficient under this procedure, as both target and nontarget items would be familiar (Herron \& Rugg, 2003b; Rugg, Henson, \& Robb, 2003; Wilding \& Rugg, 1997). Thus, participants must discriminate one class of studied items from another to succeed in this task, presumably by adopting a retrieval orientation targeted toward the appropriate class of items (Allan, Wilding, \& Rugg, 1998; Herron \& Rugg, 2003a, 2003b; Herron \& Wilding, 2005; Wilding \& Rugg, 1997). Accordingly, larger retrieval orientation effects arise when memory is tested under an exclusion procedure as opposed to a simple recognition-based procedure (Johnson \& Rugg, 2006). 
Johnson and McGhee (2015) recently employed the exclusion procedure to test retrieval orientation effects to memory age, and observed more positive-going new-item waveforms over the posterior scalp when recent (30 minutes earlier) compared to remote (one week ago) items were targeted in distinct retrieval blocks. These findings indicate that individuals can flexibly adjust their retrieval orientation on the basis of memory age to target items encoded in different time points, just as they might use encoding modality or task demands as a basis for retrieval orientation in other instances.

One outstanding issue regarding the interpretation of the above findings from Johnson and McGhee (2015) is the extent to which the observed effects of orienting retrieval to memory age are confounded with differences in the difficulty of retrieving memories from different times. As discussed earlier, it is clear from behavioral studies that retrieval difficulty increases as the retention interval grows and memories become older (Gardiner \& Java, 1991; Mitchell, Brown, \& Murphy, 1990; Nadel, Campbell, \& Ryan, 2007; Squire, 1989; for reviews, see Roediger, Weinstein, \& Agarwal, 2010; Wixted, 2004). As a result, it is possible that the putative orientation effects instead reflect retrieval difficulty or the effort expended to overcome it (Buckner, Koutstaal, Schacter, Wagner, \& Rosen, 1998; Donaldson, Allan, \& Wilding, 2002; Dzulkifli et al., 2004; Robb \& Rugg, 2002; Rugg \& Wilding, 2000). Johnson and McGhee (2015) attempted to reduce the influence of differences in retrieval difficulty in their results using two post hoc methods: trials were matched across targeted test blocks according to their response times (RTs), and participants were segregated into groups that showed larger versus smaller differences in difficulty across the blocks. Although the retrieval orientation effects remained largely unchanged, the possibility remains that other 
differences were not captured by these correction methods and thus were not effectively ruled out. An alternative method for dissociating the effects of retrieval orientation from difficulty would be to directly manipulate the two effects simultaneously, and in doing so, match the difficulty across orientation conditions (e.g., Robb \& Rugg, 2002). The first experiment (Experiment 1) presented here adopted this strategy by crossing a manipulation of the number of item repetitions during encoding with the remote and recent conditions. Persistent differences in the new-item ERPs in this context would provide compelling support for the role of retrieval orientation in targeting memories from different time points.

A second issue surrounding the effects of retrieval orientation for memory age concerns the nature of processing differences between these different retrieval situations. More precisely, although it is becoming increasingly clear that time-related information is strategically used to target memories encoded at different times, it remains unclear how this information is being used. For the standard type of retrieval orientation study in which encoded material or task is manipulated (e.g., Dzulkifli et al., 2004; Johnson \& Rugg, 2006b), one proposal is that orienting processes are employed to increase the match in features between the retrieval cue and the targeted memories (see Herron \& Wilding, 2004; Hornberger et al., 2004; Rugg \& Wilding, 2000). For example, orthographic features might be highlighted for word targets, whereas perceptual features might be highlighted for picture targets. However, it is unclear how this interpretation would apply to orientation effects for memory age, as such effects arise even when target items were encoded in the same physical format, and under the same task demands (Johnson \& McGhee, 2015). An alternative hypothesis is that participants use other 
characteristics about the memory traces to guide retrieval processing when targeting items from a specific time. These characteristics could include a number of features that could reasonably be expected to vary with memory age, such as the relative strength of the items (e.g., Hinrichs,1970; Hintzman, 2005; Wickelgren, 1974), the degree to which they match current self-referential processes or other contextual features (e.g., Hintzman, Block, \& Summers,1973; Hintzman, 2002; Howard \& Kahana, 2002), or the amount or specificity of qualitative information that is expected to be retrieved (e.g., Piefke, Weiss, Zilles, Markowitsch, \& Fink, 2003; Rudoy et al., 2009; Talamini \& Gorree, 2012; for a review, see Moscovitch, Cabeza, Winocur, \& Nadel, 2016). The second experiment (Experiment 2) presented here directly tests the idea that the orientation effects reflect the specificity of retrieval. 


\section{Chapter 2}

\section{EXPERIMENT 1}

\subsection{INTRODUCTION}

In Experiment 1, we tested for a distinction between retrieval orientation and difficulty by introducing an experimental manipulation of the number of times each item was presented within the remote and recent encoding sessions. This manipulation was intended not only to match behavioral performance across the later memory tests that targeted remote and recent items, but also to allow for dissociation of the difficulty and orientation ERP effects (e.g., Robb \& Rugg, 2002). As in Johnson and McGhee (2015), participants completed two encoding sessions separated by one week. In each session, pleasantness ratings were made for a series of pictures of common objects. Pictures were used in the current experiment, instead of the words used by Johnson and McGhee, to help improve performance on retrieving items from the remote (one week ago) encoding session. In each encoding session, half of the items were presented only once, while the other half were presented four times (all randomly intermixed). The memory retrieval phase followed completion of the second encoding session, and used an exclusion task procedure (Jacoby, 1991, 1998; Jacoby et al., 1993) to target items from each combination of the remote/recent and item repetition conditions. To isolate retrieval orientation effects from potential confounding effects due to the remote-recent manipulation (such as differential rates of retrieval success or different information being 
retrieved, as previously discussed), the ERP analyses focused primarily on correctly rejected new items. Finally, to evaluate whether the item repetition manipulation successfully matched retrieval difficulty across the encoding sessions, the key contrast involved the test block targeting remote items that were studied four times and that which targeted recent items studied once.

\subsection{METHOD}

\subsubsection{Participants}

Twenty-two students from the University of Missouri (MU) participated for partial fulfillment of course credit. All participants were screened to ensure that they were right-handed, native-English speakers, with normal or corrected vision, and no history of neurological disorders. Data from five participants were excluded from all analyses: three of these participants did not return to complete the second phase of the experiment, and two participants were excluded due to technical errors in stimulus presentation. The final sample of 17 participants (11 males) were 18 to 22 years of age $(M=19)$. Informed consent was obtained in accordance with the MU Institutional Review Board.

\subsubsection{Materials}

Stimuli were drawn from a pool of 400 pictures of everyday objects. For each participant, pictures were randomly selected from the pool to construct two study lists consisting of 100 items each. One list was assigned to the Remote condition and was presented during a participant's first visit to the laboratory; the other list was assigned to the Recent condition and was presented at the beginning of the participant's second visit. Within each study list, half of the items appeared only once, while items from the remaining half each appeared four times. Four test lists were constructed for each 
participant by randomly drawing 30 target items from the corresponding repetition condition in the appropriate study list, along with 20 nontarget items from the opposing study list (drawn randomly and equally across repetition condition), and 30 items randomly selected from the stimulus pool to serve as new items. Pictures were presented in 16-color format on a solid gray frame at the center of the black background of a 24inch widescreen LCD monitor (cropped to 1024 x 768 resolution). All instructions, response options, and fixation crosses were displayed in white uppercase 36-point Arial font. The monitor was viewed at a distance of approximately $1 \mathrm{~m}$, with maximum visual angles of $0.7^{\circ}$ for letter height, $4.1^{\circ}$ for word length, $3.1^{\circ} \times 3.1^{\circ}$ for picture size, and $4.6^{\circ}$ x $4.6^{\circ}$ for the gray frame. The Cogent 2000 toolbox (http://www.vislab.ucl.ac.uk) was used to control stimulus presentation in MATLAB (The MathWorks, Natick, MA).

\subsubsection{Design and Procedure}

For the first visit to the laboratory, participants completed the Remote study phase, which lasted about 30 minutes. Participants returned approximately one week (6 to 8 days) later to complete the Recent study phase, followed by preparation of the EEG cap and electrodes, the targeted test phase, and then the final test phase. The second visit lasted about two hours.

For each study phase, participants were seated in front of a computer monitor and instructed to make pleasantness ratings for a series of object pictures. The right index through little fingers were used to press keys on the keyboard corresponding to "very pleasant", "somewhat pleasant", "somewhat unpleasant", and "very unpleasant", respectively. During the instructions for the study phases, participants were informed that some of the pictures would occasionally repeat, and it was emphasized that their 
responses should be based on how they felt in that moment rather than thinking back to how they previously responded. Items presented either once or four times were randomly intermixed throughout each study phase. For the Recent study phase, participants were additionally informed that the upcoming pictures had not been presented during the previous (Remote) list. Each picture was presented for $3 \mathrm{~s}$, with a central fixation cross displayed for a random interval between 1 and $2 \mathrm{~s}$ until the next picture. Participants were instructed to respond before each picture disappeared. A short break occurred at the midpoint of each study phase.

Following completion of the Recent study phase, EEG preparation lasted approximately 30 minutes. In order to minimize any differences in encoding strategies across the two study phases, participants were kept naïve to the nature of the test phase until immediately before its start. The test phase was divided into four blocks, each of which employed an exclusion task procedure to selectively target pictures from one of the four study conditions (Remote vs. Recent items presented one vs. four times). For example, in the block targeting Remote items presented one time, nontargets were Recent items presented one time. Targeted pictures required a positive response made with the index finger of the right hand, whereas nontargeted and new pictures required a negative response made with the right middle finger. Each picture was presented for $3 \mathrm{~s}$, followed by a central fixation cross displayed for a random interval between 1 and $2 \mathrm{~s}$ until the next picture appeared. Participants were instructed to respond before each picture disappeared. Test blocks were separated by breaks of about five minutes each, during which participants were given instructions and a short practice on the task for the upcoming block. The Remote and Recent blocks followed an ABBA order that was 
counterbalanced across participants, whereas blocks directed at items presented one vs. four times were randomized.

After completing the targeted test phase, participants undertook a final test phase that assessed memory for new items presented during the targeted test blocks. Such memory tests have been used in other contexts to examine the effects of orienting during the targeted test blocks on subsequent recognition of new items that appeared in those blocks, using purely behavioral measures (Jacoby et al., 2005; Shimizu \& Jacoby, 2005). Although Johnson and McGhee (2015) reported no significant findings from the final test phase in their experiment, we opted to include the final test phase in the present experiment to allow for further examination of behavioral effects of orienting during the targeted test phase. Prior to the start of the final test, participants were briefly instructed to make a positive response to pictures from the targeted test phase, and a negative response to new pictures (using the right index and middle finger, respectively). To reduce the overall length of the experiment, there was no practice version of this task. The timing of the pictures and fixation marker was the same as in the targeted test phase, and a 1-2 minute break occurred at the midpoint.

\subsubsection{EEG Acquisition, Processing, and Analysis}

EEG data were continuously recorded during the targeted test phase from 59 $\mathrm{Ag} / \mathrm{AgCl}$ sintered ring electrodes embedded in an elastic cap (Easycap, Herrsching, Germany; http://www.easycap.de), using a BrainAmp Standard system (BrainVision LLC; Durham, NC; http://www.brainvision.com). Electrode placement was based on the extended 10-20 system (Easycap montage no. 11) and included the following sites: Fpz, Fp1/2, AFz, AF3/4/7/8, Fz, F1-F8, FC1-FC6, FT7/8, Cz, C1-C6, T7/8, CPz, CP1-CP6, 
TP7/8, Pz, P1-P8, POz, PO3/4/7/8, and O1/2. The reference and ground electrodes were placed at the FCz and FT10 locations, respectively. EOG data were recorded from electrodes placed below the left eye and on the outer canthi of each eye. Two additional electrodes were placed on the mastoids for offline re-referencing. Impedances were adjusted to below $5 \mathrm{k} \Omega$ before starting the test phase. The data were recorded at a sampling rate of $1 \mathrm{kHz}$ with an amplifier bandwidth of $0.01-100 \mathrm{~Hz}$.

Offline processing of the EEG data was implemented with the EEGLAB (Delorme \& Makeig, 2004; http://sccn.ucsd.edu/eeglab/) toolbox in MATLAB. The continuous data were bandpass filtered from .05 to $20 \mathrm{~Hz}$, down-sampled to $200 \mathrm{~Hz}$, and re-referenced to the linked mastoids. The data were then epoched from -200 to $2000 \mathrm{~ms}$ relative to test item onset, and baseline-corrected across the pre-stimulus period, to form ERPs. Independent components analysis (ICA) was used to identify components corresponding to artifacts (e.g., eye movements, blinks, and muscle activity) that were then manually rejected on the basis of their scalp topography and power spectra. The SASICA toolbox (Chaumon, Bishop, \& Busch, 2015; https://github.com/dnacombo/SASICA) was also used for guidance on artifactual components. The ICA procedure was applied to all blocks of the test phase together to prevent any bias of removing components for one condition versus another. Finally, epochs were rejected if the signal exceeded $\pm 100 \mu \mathrm{V}$ from baseline. The mean numbers of remaining new-item trials contributing to the averaged ERPs were 24 (range: 19-30), 28 (range: 25-30), 29 (range: 22-30), and 28 (range: 26-30) for the Remote-1x, Remote4x, Recent-1x, and Recent-4x conditions, respectively. 
Permutation-based analyses were performed on the averaged ERPs to identify cluster-corrected significance values separately for the time and spatial domains (Maris \& Oostenveld, 2007). Each of these analyses comprised 10,000 random permutations of the four condition labels for each subject, using only the data for correctly rejected new items, with the resulting data submitted to the appropriate statistical test (i.e., a paired $t$ test). For the time domain, the ERP data were tested ( $p<.05$, two-tailed) at each 5-ms time point during the post-stimulus portion of the recording epoch. For each permutation, the maximum number of consecutive intervals was noted separately for positive and negative differences. For the spatial domain, the data were collapsed over the predetermined latency periods from prior studies (e.g., Dzulkifili \& Wilding, 2005; Johnson \& McGhee, 2015; Johnson \& Rugg, 2006a, 2006b; Roberts et al., 2014). Electrodes were defined as neighbors based on their adjacency in the Easycap montage 11. The maximum number of significant clustered electrodes were identified for each permutation, again separately for positive and negative differences. For each of these methods, the actual (non-shuffled) results were compared against the critical cluster sizes defined by the 2.5 and 97.5 percentiles of the distribution of permuted statistical values.

\subsection{RESULTS}

\subsubsection{Behavioral results}

The proportions of correct responses and associated RTs from the targeted test phase are provided in Table 1 . These measures were separately submitted to three-way ANOVAs that included factors of item type (target, nontarget, or new), study week (remote or recent), and study repetition (1x or $4 \mathrm{x})$. The ANOVA of the proportions gave rise to significant main effects of item type $(F(1.1,17.9)=27.05, p<.001$; degrees of 
freedom for this and subsequent effects were Greenhouse-Geisser adjusted when appropriate), study week $(F(1,16)=72.21, p<.001)$, and study repetition $(F(1,16)=$ $86.60, p<.001)$. These effects were qualified by significant two-way interactions of item type $\times$ study week $(F(1.4,21.7)=29.89, p<.001)$, item type $\times$ study repetition $(F(1.4$, 23) $=9.40, p<.01)$, and study week $\times$ study repetition $(F(1,16)=25.73, p<.001)$, as well as the three-way interaction $(F(1.7,27)=9.99, p<.01)$.

To understand the nature of the three-way interaction described above, follow up two-way ANOVAs of the correct response proportions, including factors of study week and study repetition, were conducted for each item type. For targets, the ANOVA gave rise to significant main effects of study week $(F(1,16)=50.03, p<.001)$ and study repetition $(F(1,16)=48.90, p<.001)$ and a significant interaction $(F(1,16)=17.24, p<$ .01). The interaction can be described as the additional repetitions increasing correct responses for remote items more than for recent items (see Table 1). For nontargets, the ANOVA resulted in only a main effect of study repetition $(F(1,16)=16.02, p<.01)$, such that the correct response proportion was higher for the $4 \mathrm{x}$ than the $1 \mathrm{x}$ condition. Finally, the ANOVA of the new item proportions revealed significant main effects of study week $(F(1,16)=37.77, p<.001)$ and study repetition $(F(1,16)=12.92, p<.01)$ and a significant interaction $(F(1,16)=34.70, p<.001)$. As with the targets, the study repetition manipulation appeared to increase correct responses to new items to a greater degree when the remote list was targeted compared to when the recent list was targeted. Because the main purpose of the repetition manipulation was to equate performance for new items across the remote and recent tests, we also directly contrasted the Remote- $4 \mathrm{x}$ 
and Recent-1x conditions. The correct response proportions for these two conditions were statistically equivalent $(t(16)=.59, p=.56)$.

The three-way ANOVA of RTs associated with correct responses revealed significant main effects of item type $(F(1.4,21.7)=30.40, p<.001)$, study week $(F(1,16)$ $=19.08, p<.001)$, and study repetition $(F(1,16)=11.97, p<.01)$, as well as a significant item type $\times$ study week interaction $(F(1.9,30.7)=18.08, p<.001)$. A followup two-way ANOVA of target RTs gave rise to significant main effects of study week $(F(1,16)=36.43, p<.001)$ and repetition $(F(1,16)=9.35, p<.01)$, such that RTs were faster for the recent than remote condition and faster for the $4 \mathrm{x}$ than $1 \mathrm{x}$ condition. The ANOVA of nontarget RTs revealed no significant effects. The ANOVA of the RTs to new items gave rise to significant main effects of study week $(F(1,16)=14.69, p<.01)$ and repetition $(F(1,16)=13.91, p<.01)$, reflecting a pattern of effects similar to that for targets: faster RTs for the recent and 4x conditions. Contrasting new-item RTs for the two main conditions of interest-Remote- $4 \mathrm{x}$ and Recent- $1 \mathrm{x}$ - - indicated no significant difference $(t(16)=.70, p=.49)$.

Finally, to evaluate the consequences of adopting different retrieval orientations on behavioral performance (e.g., Jacoby et al., 2005; Shimizu \& Jacoby, 2005), we turned our analyses to the final test phase. The proportions of correct responses and associated RTs from this phase are provided in Table 2 . Since we were mainly interested in the responses to different types of old items (note that these were the new items in the previous test phase), the correct proportions and RTs were submitted to two-way ANOVAs that included factors of study week (remote, recent) and study repetition (1x, 4x). The ANOVAs yielded no significant effects for either of the behavioral performance 
measures. Pairwise comparisons of the data for the two conditions of primary interestitems presented in the Remote- $4 \mathrm{x}$ and Recent- $1 \mathrm{x}$ blocks of the previous test phase-also yield no significant effects (proportions: $t(16)=1.50, p=.15$; RTs: $t(16)=.79, p=.44$ ). When collapsing across the old items from each type of previous orientation test, the proportions of correct responses to old and new items were equivalent $(t(16)=1.06, p=$ $.31)$, whereas responses were faster for old than new items $(t(16)=3.04, p<.01)$.

\subsubsection{ERP results}

Analysis of the EEG results first proceeded by testing the retrieval orientation effect for remote vs. recent memories, while controlling for difficulty via the repetition manipulation at encoding. This analysis employed latency periods throughout the retrieval epochs and an electrode montage that has been previously shown to identify retrieval orientation effects (e.g., Dzulkifli \& Wilding, 2005; Johnson \& McGhee, 2015;

Johnson \& Rugg, 2006a, 2006b; Roberts et al., 2014). Next, we used random-permutation analyses to control the family-wise error rate of the retrieval orientation findings according to multiple comparisons across time and electrodes. Finally, the effects of the repetition manipulation on the EEG data at retrieval were tested in attempt to dissociate any difficulty differences from the main retrieval orientation effect of interest.

The ERPs for the correctly rejected new items were first contrasted across the Remote-4x and Recent- $1 \mathrm{x}$ test conditions to test for retrieval orientation effects while behavioral performance (and presumably, difficulty) was equated (see Table 1). The average ERPs associated with these items are shown in Figure 1 for a broadly distributed set of electrodes. As can be seen, the ERPs for the Recent-1x condition appear more positive than the Remote-4x condition over posterior scalp, beginning at about $500 \mathrm{~ms}$ 
after stimulus onset and continuing through the end of the recording epoch. To analyze these data, the mean voltage amplitudes were extracted from the montage of fifteen electrodes (F7, F3, Fz, F4, F8; T7, C3, Cz, C4, T8; P7, P3, Pz, P4, P8) in a series of six latency periods (200-500, 500-800, 800-1100, 1100-1400, 1400-1700, and 1700-2000 ms post-stimulus onset; also see Dzulkifli \& Wilding, 2005; Johnson \& McGhee, 2015; Johnson \& Rugg, 2006a, 2006b; Robb \& Rugg, 2002; Roberts et al., 2014). The data were then submitted to an ANOVA that employed factors of orienting condition (Remote-4x vs. Recent-1x), anterior/posterior chain (frontal, central, and parietal), laterality (inferior left, superior left, midline, superior right, and inferior right), and latency period. Since our hypotheses centered on the orienting effect, we report only the significant effects that included that factor. The omnibus ANOVA gave rise only to a significant two-way interaction of orienting condition and anterior/posterior chain $(F(1.7$, $27.1)=7.36, p<.01)$.

Because our predictions were that the retrieval orientation effect would take some time to onset (also see Johnson \& McGhee, 2015), we also conducted subsidiary ANOVAs for each latency period, employing the remaining factors. This analysis revealed significant two-way interactions between orienting condition and anterior/posterior chain in the second through fifth latency periods: 500-800 $\mathrm{ms}(F(1.3$, $20.7)=4.18, p<.05), 800-1100 \mathrm{~ms}(F(1.5,24.7)=9.15, p<.01), 1100-1400 \mathrm{~ms}(F(1.5$, $23.2)=7.83, p<.01)$, and $1400-1700 \mathrm{~ms}(F(1.6,26.2)=8.26, p<.01)$. To explore the differences across anterior and posterior scalp, follow-up ANOVAs were conducted within each latency period, including factors of orienting condition and laterality. Significant main effects of orienting condition were observed for the parietal electrode 
chain in the 500-800 $\mathrm{ms}(F(1,16)=6.48, p<.05), 800-1100 \mathrm{~ms}(F(1,16)=5.84, p<$ $.05)$, and $1400-1700 \mathrm{~ms}(F(1,16)=6.36, p<.05)$ periods, reflecting the more positivegoing ERPs for the Recent-1x than Remote-4x condition. No significant effects were revealed from these analyses for the frontal and central electrode chains.

To identify more precisely the time course of the effect described above - that is, irrespective of the predetermined latency periods - we collapsed the ERPs across the electrodes within each anterior/posterior chain (i.e., across the laterality factor, given that it was not associated with any significant effects). The difference waveforms (Recent-1x minus Remote-4x) for each electrode chain are shown in Figure 2A. For each time point, we tested whether the difference was significant, and the resulting time course of $t$-values across the recording epoch are shown in Figure 2B. Random permutations of the average ERPs for each subject were then carried out to determine the number of consecutively significant time points occurred by chance (also see Section 2.2.4). As shown in Figure $2 \mathrm{~B}$, there were two clusters of time points that passed the cluster-corrected threshold of $p$ $<.05$ : one of these clusters took place from $670-800 \mathrm{~ms}$ and the other occurred from 930 $1055 \mathrm{~ms}$.

To further test the reliability of the retrieval orientation effects, we next submitted the corresponding topographic scalp maps to permutation analyses. For these analyses, the ERP differences for correctly rejected new items in the Recent-1x and Remote-4x conditions were averaged within the predetermined latency periods at each electrode. The resulting topographic maps are displayed in Figure 3. As is highlighted in the figure, significant clusters of adjacent electrodes were evident over right posterior scalp in the 200-500 ms latency period and over bilateral posterior scalp (although maximal over the 
right) in the 500-800 ms latency period. Although comparable posterior differences seemed to be present during some of the later periods (800-1100 and 1400-1700 ms), these differences failed to pass the cluster correction procedure due to the midline posterior electrodes not being individually significant (and, as a result, dividing the effects into two smaller clusters).

Finally, effects of the repetition manipulation during encoding were tested on the new-item ERPs in order to potentially dissociate such effects from those described above. For this analysis, we focused on repetition effects within the remote memory tests, which resulted in the largest behavioral differences according to this manipulation (see Table 1). The ERPs for the Remote-4x and Remote-1x conditions at the 15 electrodes used earlier are shown in Figure 4. ANOVA of these data included the latency period and electrode factors previously used, along with a factor for the two repetition conditions. Because we were interested only in the repetition manipulation for this set of analyses, we report only the significant effects that included that factor. The omnibus ANOVA revealed no significant effects, so we next tested for remote repetition effects within each latency period. The ANOVAs for each period gave rise only to a significant three-way interaction between repetition, anterior/posterior chain, and laterality, which was restricted to the $200-500$ ms period $(F(3.9,61.9)=3.31, p<.05)$. Pair-wise $t$-tests at each electrode within this latency period revealed significant differences only at the P7 $(t(16)=2.65, p<$ $.05)$ and P8 $(t(16)=3.35, p<.01)$ electrodes. Notably, these differences took the form of more positive-going ERPs for the Remote-1x condition compared to the Remote-4x condition-opposite to what we anticipated, given that the more difficult condition in our 
original comparison for retrieval orientation was more negative (see Johnson \& McGhee, 2015).

One possible explanation for our observation of an unpredicted pattern of repetition (i.e., difficulty) effects in the above analysis is that the effects may have occurred outside the range of the montage of 15 electrodes analyzed. To address this, we repeated the ANOVA of the repetition effect within remote tests, this time using all 59 electrodes (characterized by a single factor). The omnibus ANOVA revealed no significant effects, as did the subsidiary ANOVA for each latency period. (The maximum $F$-value for the condition $\times$ electrode interaction occurred for the $800-1100 \mathrm{~ms}$ interval:

$F(3.1,49.5)=1.54, p=.215$.) For comparison, we also conducted similar analyses of the retrieval orientation effect (Recent-1x vs. Remote-4x) using all 59 electrodes. The ANOVA yielded a significant orienting condition $\times$ electrode interaction $(F(4.5,72.2)=$ $2.65, p<.05)$. Subsidiary ANOVAs of the orienting effects in each latency period additionally gave rise to significant interactions during $800-1100 \mathrm{~ms}(F(4.9,78.7)=3.18$, $p<.05), 1100-1400 \mathrm{~ms}(F(4.4,71.0)=3.06, p<.05)$, and $1400-1700 \mathrm{~ms}(F(3.8,61.4)=$ $3.39, p<.05)$.

\subsection{SUMMARY}

The purpose of Experiment 1 was to investigate the effects of orienting retrieval processing to remote and recent memories while controlling for the difficulty of the retrieval task. In comparison with our previous study that examined the influence of difficulty on retrieval orientation in a post-hoc manner (Johnson \& McGhee, 2015), difficulty was addressed directly in the present experiment with a manipulation of item repetition - one versus four presentations — at the time of encoding. This manipulation 
successfully resulted in two conditions in which behavioral performance was equated for correctly rejected new items. The main result was that differences were observed in the new-item ERPs from these two equated conditions. Consistent with our previous findings, these differences took the form of more positive-going ERPs for the recent test condition. Additionally, these effects were similarly maximal over posterior electrodes and onset by about $500 \mathrm{~ms}$ after the presentation of test items. We interpret these findings as further support for the idea that the age of a memory trace can be used to strategically orient the processing of retrieval cues (Johnson \& McGhee, 2015; also see Roberts et al., 2014). The potential consequences of this differential processing, however, revealed no significant effects on behavior in the final test phase (for similar findings, see Johnson \& McGhee, 2015).

In addition to the repetition manipulation providing conditions of equated memory performance, it also allowed for investigation of the ERP effects associated with different levels of retrieval difficulty. Comparison of the repetition effect across the tests targeting retrieval of remote items gave rise to a posterior difference which, while having a similar topographic distribution as the retrieval orientation effect, was dissociable in two respects. First, it occurred earlier than the retrieval orientation effect, such that it was restricted to the 200-500 ms interval whereas the retrieval orientation effect did not onset until $500 \mathrm{~ms}$ post-stimulus onset. Second, it took the opposite form of the retrieval orientation difference; whereas the ERPs for the more difficult condition (i.e., Remote4x; also see Johnson \& McGhee, 2015) in the retrieval orientation comparison was more negative-going, those same ERPs were more positive-going in the repetition contrast. 
Together, these distinctions indicate that the effect of orienting to memory age is not predominantly driven by differences in retrieval difficulty. 


\section{Table 1}

Mean proportions of correct responses and corresponding RTs during the targeted memory tests in Experiment 1

\begin{tabular}{|l|c|c|c|c|}
\hline \multicolumn{5}{|c|}{ Study condition } \\
\hline Test item type & Remote, 1x & Remote, 4x & Recent, 1x & Recent, 4x \\
\hline Proportions & $.52(.06)$ & $.73(.05)$ & $.83(.04)$ & $.93(.02)$ \\
\hline Target & $.94(.02)$ & $.97(.01)$ & $.89(.02)$ & $.97(.01)$ \\
\hline Nontarget & $.85(.02)$ & $.95(.01)$ & $.97(.02)$ & $.98(.01)$ \\
\hline New & $1459(79)$ & $1305(60)$ & $1215(49)$ & $1104(42)$ \\
\hline$R T s$ & $1144(74)$ & $1104(50)$ & $1121(54)$ & $1083(56)$ \\
\hline Target & $1231(84)$ & $1089(60)$ & $1065(51)$ & $971(51)$ \\
\hline Nontarget
\end{tabular}

Note: SEMs are in parentheses. RTs are in milliseconds. 


\section{Table 2}

Mean proportions of correct responses and corresponding RTs during the final test phase in Experiment 1

\begin{tabular}{|l|c|c|}
\hline Final test item type & Proportions & RTs \\
\hline Old & $.84(.02)$ & $1091(23)$ \\
\hline Remote, $\mathbf{1} \boldsymbol{x}$ & $.83(.05)$ & $1095(51)$ \\
\hline Remote, $\mathbf{4} \boldsymbol{x}$ & $.82(.04)$ & $1088(45)$ \\
\hline Recent, $\mathbf{1} \boldsymbol{x}$ & $.85(.04)$ & $1072(45)$ \\
\hline Recent, $\mathbf{4} \boldsymbol{x}$ & $.85(.03)$ & $1109(43)$ \\
\hline New & $.88(.02)$ & $1196(52)$ \\
\hline
\end{tabular}

Note: SEMs are in parentheses. RTs are in milliseconds. 


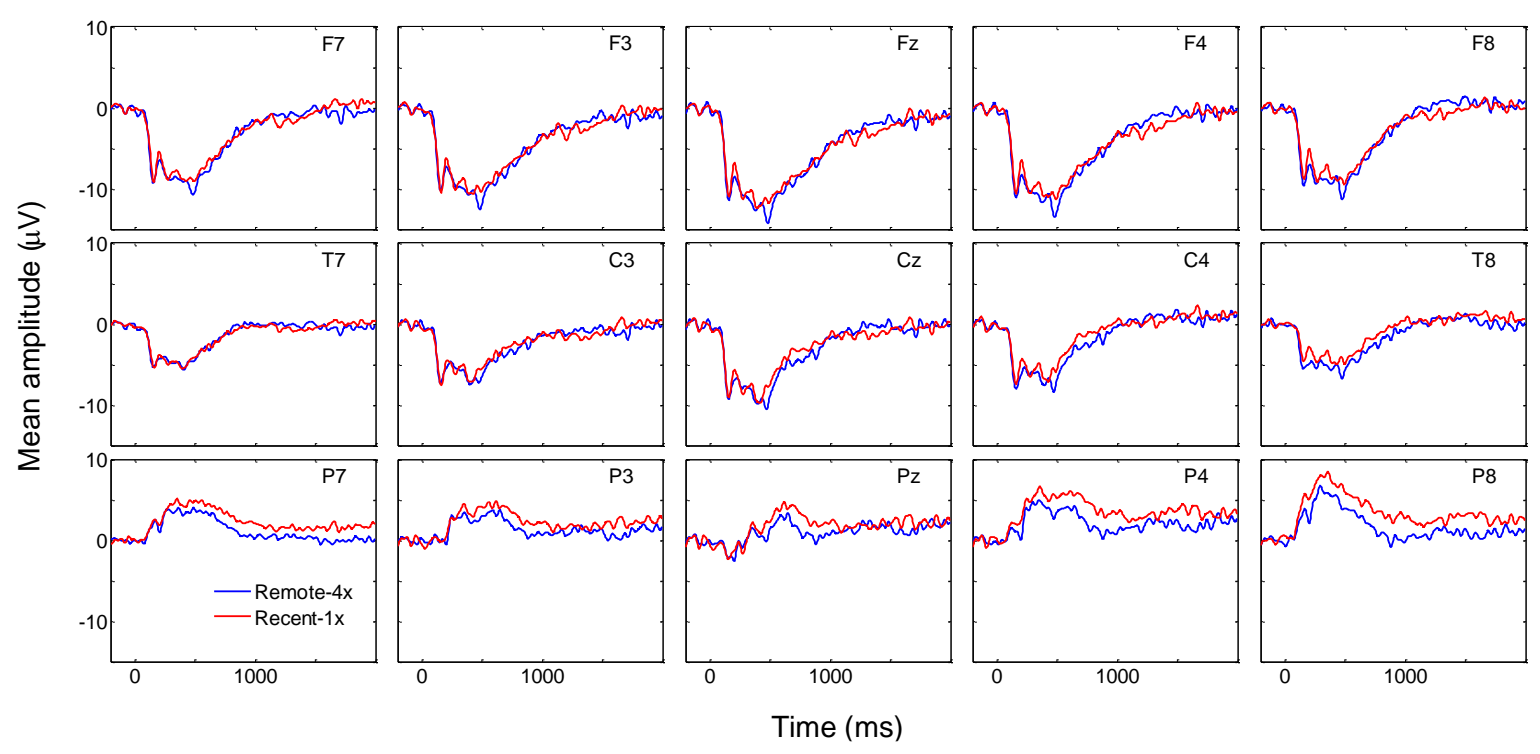

Figure 1. Mean ERPs during Remote-4x and Recent-1x orienting in Experiment

1. Grand-average ERPs elicited by correctly rejected new items, according to whether Remote-4x (blue) or Recent-1x (red) memories were targeted. The electrodes corresponding to the ERPs are indicated in the upper right corner of each plot. 
A

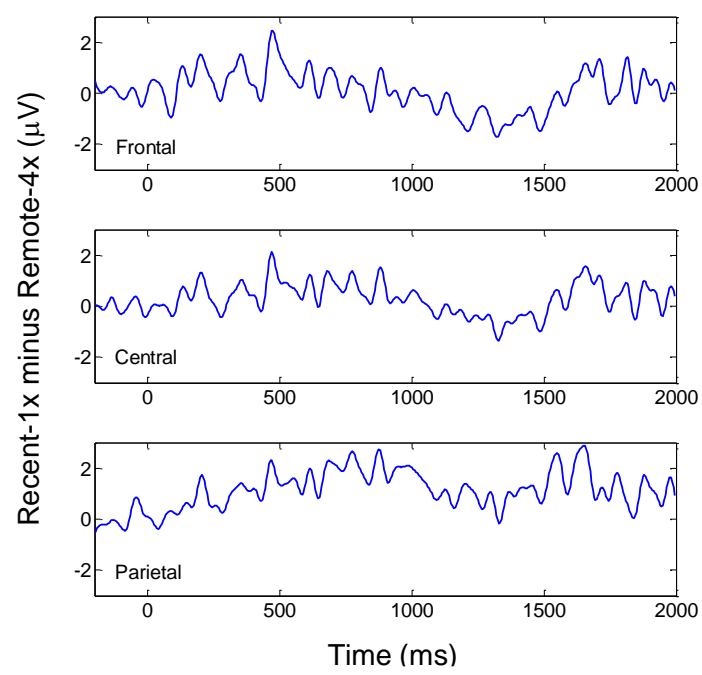

$\mathrm{B}$

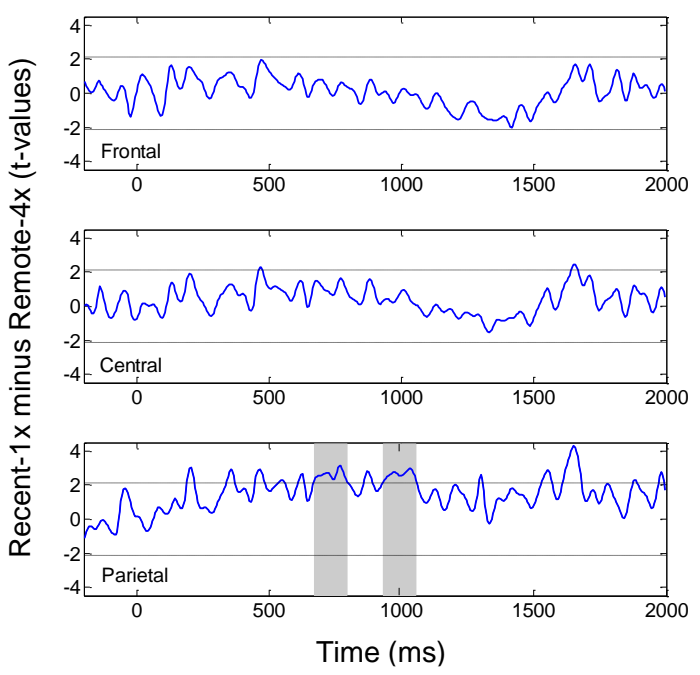

Figure 2. Difference waveforms for the Recent-1x minus Remote-4x contrast in Experiment 1. A) Difference waveforms between the ERPs to correctly rejected new items in the Recent-1x and Remote-4x tests, plotted separately for each anterior/posterior chain: frontal (top panel), central (middle panel), and parietal chain (bottom panel). B) Corresponding time courses of $t$-values across the recording epoch for the ERP contrasts depicted in the left panel. The dotted lines represent the critical $t$-value (uncorrected), and the gray shaded panels indicate the clusters of time points that passed the cluster-corrected threshold of $p<.05$. 


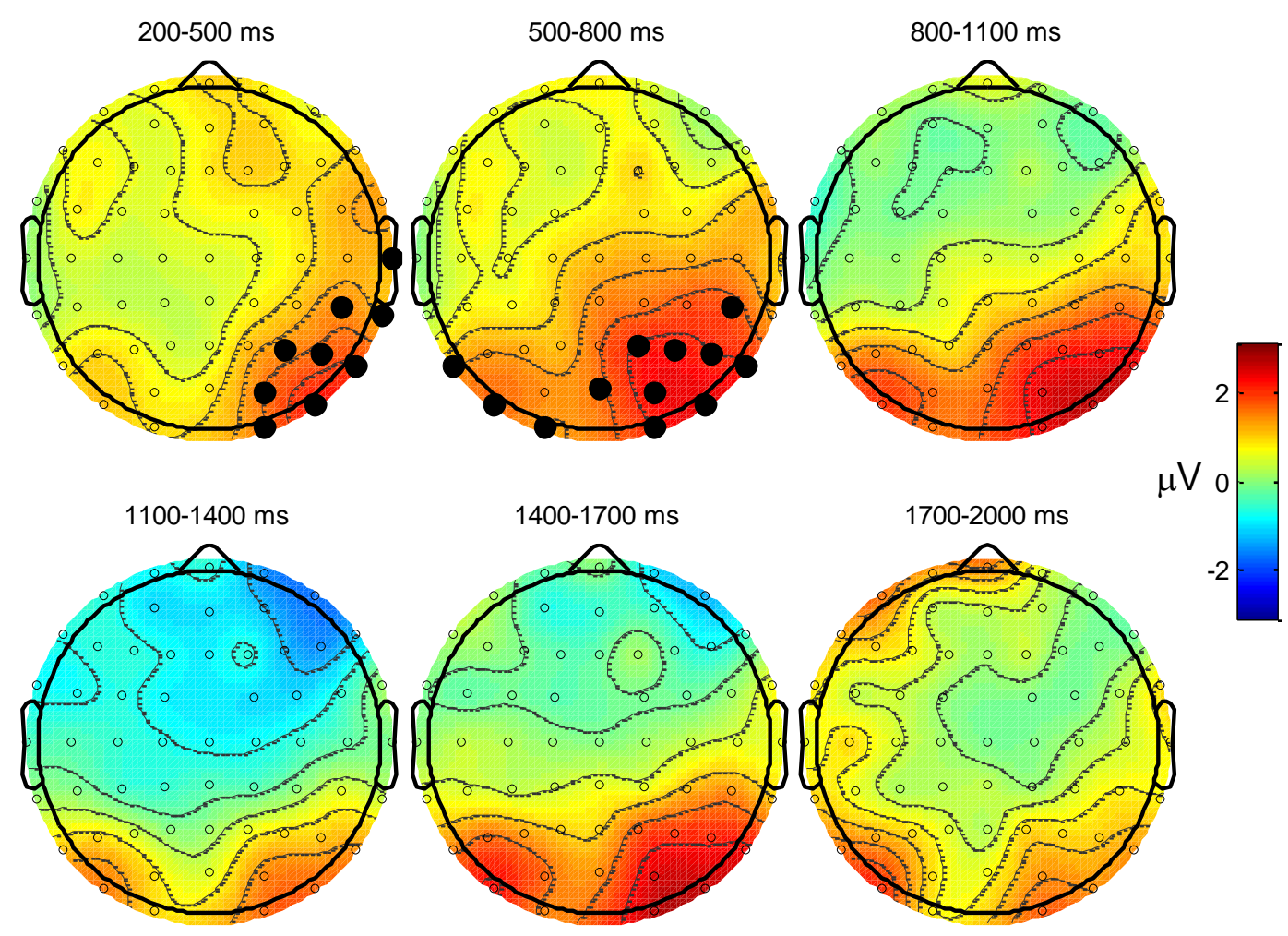

Figure 3. Topographic maps for the Recent-1x minus Remote-4x contrast in

Experiment 1. Topographic maps resulting from the contrast of Recent-1x minus Remote4x orienting conditions within each predetermined latency period. Electrodes comprising significant clusters associated with the retrieval orientation effect are indicated with larger, filled circles. 


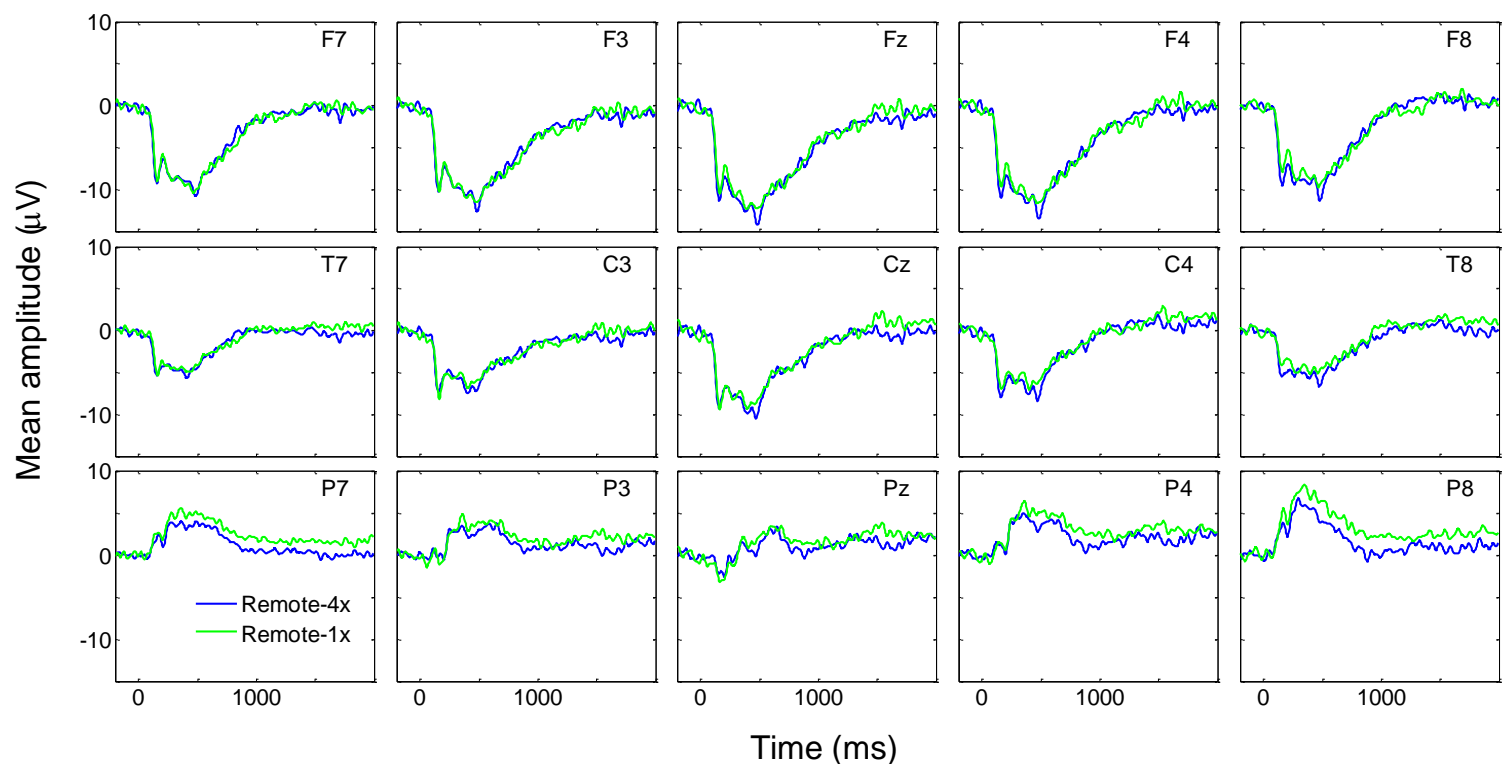

Figure 4. Mean ERPs during Remote-4x and Remote-1x orienting in Experiment

1. Grand-average ERPs elicited by correctly rejected new items, according to whether Remote-4x (blue) or Remote-1x (green) memories were targeted. The electrodes corresponding to the ERPs are indicated in the upper right corner of each plot. 


\section{Chapter 3}

\section{EXPERIMENT 2}

\subsection{INTRODUCTION}

As described in the General Introduction, Experiment 2 is an attempt to fill a void in the functional interpretation of retrieval orientation effects that differ according to memory age. One interpretation of these findings is that individuals differentially expect to recover episodic details for remote as opposed to recent memories (Johnson \& McGhee, 2015) and, as a result, they might alter their retrieval processing strategies when targeting memories from these distinct time points. Targeting remote memories, for instance, might elicit processes aimed at recovering fewer details or memories that are less specific (i.e., less vivid or more gist-based). By contrast, targeting recent memories might engage processes expected to access a greater number of or more-specific details. The present study tested this hypothesis by experimentally manipulating the specificity of participants' cognitive processing demands prior to memory retrieval.

Experiment 2 implemented a novel, computer-based adaptation of a technique that has recently been shown to improve the specificity of memory retrieval in young (Madore \& Schacter, 2016) and older adults (Madore, Gaesser, \& Schacter, 2014). This technique, called episodic specificity induction, involved having participants watch a video of an everyday scenario and then complete an adaptation of the Cognitive Interview (Fisher \& Geiselman, 1992) where they were prompted to elaborate on specific details remembered from the video (Madore, Addis, \& Schacter, 2015; Madore et al., 
2014; Madore \& Schacter, 2014; Madore, Szpunar, Addis, \& Schacter, 2016). When memory was tested immediately after the induction phase and with different materials (i.e., unrelated to the induction), memory performance was enhanced relative to following a general control induction (Madore et al., 2014; Madore \& Schacter, 2016). The improvement in memory performance following the episodic specificity induction has been attributed to carryover of the cognitive state engaged during the Cognitive Interview, which presumably enhances specific episodic processing (Madore et al., 2014). In the current experiment, we developed a computer-based task designed to emulate the episodic-specificity induction phase. Participants were presented with a series of words denoting common objects, and made ratings for each word according to how vividly they could imagine the object in a particular scenario. The general versus specific manipulation of this induction phase was carried out by having participants imagine either one or three scenarios, respectively, for each word. We predicted that further imagining would translate to more specific episodic processing, producing detectable changes in our observed effects of retrieval orientation to memory age. As in Experiment 1, participants made pleasantness judgments for a series of pictures presented during two encoding sessions that were separated by one week. Given that behavioral performance was equated in the previous experiment for retrieval of remote items presented four times and recent items presented once, and to save time during the experimental session, we only presented encoding items in those two conditions (as opposed to fully crossing repetition with remoteness). Following the second encoding session, participants completed a series of task cycles consisting of our variation of the episodic-specificity induction phase followed by a targeted retrieval 
block. The main focus of our analyses was to examine the concomitant changes in behavioral performance and retrieval orientation ERP effects to memory age as a result of the level of specificity induction. If the retrieval of remote items is driven by naturally orienting toward the recovery of less specific (gist-based) memories, we predicted that inducing participants into more specific episodic processing should improve memory performance for remote items and reduce the corresponding orientation-related ERP differences relative to recent item retrieval.

\subsection{METHOD}

\subsubsection{Participants}

Twenty-three MU students participated for partial fulfillment of course credit. All participants were screened to ensure that they were right-handed, native-English speakers, with normal or corrected vision, and no history of neurological disorders. None of the participants took part in Experiment 1. The behavioral and EEG data from five participants were excluded from all analyses. Two of these participants did not return to complete the second phase of the experiment, one participant was excluded due to behavioral performance that was more than two standard deviations below the mean, one was excluded due to technical errors in stimulus presentation, and a final participant was excluded due to excessive artifact in the EEG. The final sample of 18 participants (13 males) were 18 to 21 years of age $(M=19)$. Informed consent was obtained in accordance with the MU Institutional Review Board.

\subsubsection{Materials}

Stimuli were selected from the same pool of 400 pictures used in Experiment 1 and additionally included the common names of those pictures. For each participant, 
pictures were randomly assigned to two study lists—-Remote and Recent—consisting of 96 items each. Sixty of the pictures from each list were presented again as targets in the test phase, whereas the remaining 36 from each list served as nontargets. A list of 120 pictures were randomly selected from the pool to appear as new items during the test. An additional set of 60 pictures from the pool were assigned to appear in word-form during the induction phase. The word and picture stimuli, instructions, response options, and fixations crosses were displayed in the same format and on the same type of monitor as in Experiment 1 . The viewing distance and maximum visual angles were also the same as in Experiment 1. The Cogent 2000 toolbox (http://www.vislab.ucl.ac.uk) was used to control stimulus presentation in MATLAB (The MathWorks, Natick, MA).

\subsubsection{Design and Procedure}

The Remote study phase took place during the first visit to the laboratory, lasting approximately 30 minutes. Participants returned about one week (6 to 8 days) later for the Recent study phase, followed by EEG preparation, and then several cycles of the induction and test blocks. The second visit lasted about 2.5 hours.

For each study phase, participants made pleasantness ratings for a series of centrally-presented pictures, as in Experiment 1. Given that behavioral performance on the memory test was nearly equated in Experiment 1 for the Remote study items presented four times and the Recent items presented once, the study phases of the current experiment focused solely on those two conditions to maximize the number of observations. For the Remote study phase, four random orderings of the list were constructed and presented with short, intervening breaks. As before, participants were instructed to avoid referring back to their previous response for a given picture. For the 
Recent study phase, items were presented once with the further instruction that the pictures were not seen in the previous phase. Ratings were made with the left index through little fingers, corresponding to "very pleasant", "somewhat pleasant", "somewhat unpleasant", and "very unpleasant," respectively. Each study item was displayed for $3 \mathrm{~s}$, during which participants were instructed to respond, and then a fixation cross appeared for 1 to 2 seconds until the next picture.

Following completion of the Recent study phase, participants received instructions and practice for the induction and test phases. This was followed by preparation of the EEG cap and electrodes, which lasted for about 30 minutes. These two phases proceeded in an alternating manner for the remainder of the experiment, resulting in 12 induction-test cycles. Half of the cycles involved test phases that targeted pictures from the Remote study phase, whereas the other half targeted pictures from the Recent study phase. Each test phase consisted of 10 targets, six nontargets (from the alternative study list), and 10 new pictures. Targets required a positive key-press response with the right index finger, and nontargets and new items required a rejection response with the right middle finger. At the beginning of each test phase, an instruction screen was displayed for $5 \mathrm{~s}$ indicating which items (Remote or Recent) were about to be targeted. For each test trial, a picture was presented for $3 \mathrm{~s}$, and participants were instructed to respond during that time. Trials were separated by a central fixation cross displayed for a random interval between 1 and $2 \mathrm{~s}$.

For each type of targeted test (Remote, Recent), half of the preceding induction phases were General and the other half were Specific. General induction phases involved the presentation of five words denoting common objects, and required participants to 
imagine drawing the object denoted by each word. Specific induction phases involved the presentation of five words, three times each, with each presentation requiring one of three judgments: drawing the object, placing the object somewhere in your home, or taking a photo of the object. Participants were instructed to make a response for each item in the induction phases on a four-point scale according to the vividness with which the task was imagined, using the index through little fingers of the right hand. At the beginning of each induction phase, an instruction screen was displayed for $5 \mathrm{~s}$ indicating which type of induction (Specific or General) was about to occur. At the start of each induction trial, a fixation cross and a label indicating which judgment to perform (Draw, Home, Photo) were presented for $2 \mathrm{~s}$. Next, the word was displayed in place of the fixation cross for $6 \mathrm{~s}$, and participants were instructed to imagine the task for the duration of that time.

Following this, the word changed in color from white to green, signaling participants to make their response. Participants were allotted $2 \mathrm{~s}$ to respond, after which the next trial immediately began. The interval separating the induction and test phase of each cycle was kept at a fixed length of 5 seconds, while each induction-test cycle was followed by a short break controlled by the participant. The items in each induction phase were presented in a random order. The order of the four types of induction-test cycles (General-Remote, Specific-Remote, General-Recent, and Specific-Recent) was also randomized for each subject.

\subsubsection{EEG Acquisition, Processing, and Analysis}

The EEG acquisition, processing, and analysis procedures used for the EEG data were identical to those of Experiment 1. The mean numbers of new-item trials contributing to the averaged ERPs were 29 (range: 26-30), 28 (range: 19-30), 28 (range: 
20-30), and 29 (range: 20-30) for the Remote-General, Remote-Specific, RecentGeneral, and Recent-Specific conditions, respectively.

\subsection{RESULTS}

\subsubsection{Behavioral results}

The proportions of correct responses and associated RTs from the targeted test phases are provided in Table 3 . These measures were separately submitted to three-way ANOVAs that included factors of item type (target, nontarget, or new), study week (remote or recent), and induction type (general or specific). The ANOVA of the proportions gave rise to significant main effects of item type $(F(1.6,27.5)=4.21, p<$ $.05)$ and induction type $(F(1,17)=14.42, p<.01)$, two-way interactions of item type $\times$ induction type $(F(1.6,27.4)=3.68, p<.05)$ and study week $\times$ induction type $(F(1,17)=$ $34.38, p<.001)$, and the three-way interaction $(F(2.0,33.5)=8.82, p<.01)$. Analogous to the approach used for Experiment 1, these interactions were further investigated with follow up two-way ANOVAs (including factors of study week and induction type) for each item type. For targets, the ANOVA gave rise to a significant two-way interaction $(F(1,17)=11.39, p<.01)$, reflecting a larger effect of induction type on the recent tests $(t(17)=3.53, p<.01)$ than on the remote tests $(t(17)=.56, p=.58)$. The ANOVA for nontarget items also resulted in a significant interaction $(F(1,17)=5.81, p<.05)$ in which, as with targets, there was a larger induction effect on the recent $(t(17)=2.14, p<$ $.05)$ compared to remote tests $(t(17)=.18, p=.86)$. Finally, the ANOVA of the new item proportions revealed a significant main effect of induction type $(F(1,17)=43.00, p<$ $.001)$ and a significant two-way interaction $(F(1,17)=42.42, p<.001)$. As with the other 
item types, the effect of induction type was larger when recent items were targeted $(t(17)$ $=7.66, p<.001)$ than when remote items were targeted $(t(17)=1.48, p=.16)$.

To further understand the nature of the three-way interaction reported above, subsidiary ANOVAs were conducted on the correct response proportions for items from each study week. These ANOVAs employed the remaining factors of item type (target, nontarget, or new) and induction type (general or specific). The interaction was significant only for the recent week tests $(F(2,34)=11.78, p<.001)$, indicating that the largest induction effect was evident for new items. In addition, there was a significant main effect of induction for the recent week tests $(F(1,17)=29.07, p<.001)$ and a significant main effect of item type for the remote week tests $(F(1.7,29.0)=5.18, p<$ $.04)$.

To facilitate comparison of the results of this experiment with those of Experiment 1, we performed a series of additional pairwise $t$-tests on the correct response proportions for new items across the study week manipulation. First, the correct rejection rates were compared within each type of induction. Whereas following the specific induction, performance was higher for the recent compared to remote tests $(t(17)=2.33$, $p<.05)$, the opposite pattern was observed following the general induction $(t(17)=4.69$, $p<.001)$. (Recall that the interaction was also significant, as reported above.) The correct rejection rate for the recent test following the general induction was also lower than that for the remote test following the specific induction $(t(17)=3.85, p<.01)$.

The omnibus ANOVA of RTs associated with correct responses, employing the factors of item type, study week, and induction type, revealed only a significant item type $\times$ study week interaction $(F(2.0,33.9)=9.13, p<.01)$. Follow-up two-way ANOVAs 
were conducted on the RTs for each item type, as was done for the proportion data. These ANOVAs gave rise to significant main effects of study week for the target $(F(1,17)=$ $13.74, p<.01)$ and nontarget items $(F(1,17)=5.66, p<.05)$. These effects were in opposite directions, however: Whereas target RTs were longer in the remote compared to recent tests, nontarget RTs were longer during the recent tests. The ANOVA of new-item RTs gave rise to no significant effects. Finally, the planned comparisons of new-item RTs across the study week manipulation also failed to reveal any significant differences, either within induction type, or between the recent test following the general induction and the remote test following the specific induction (maximum $t(17)=.93, p=.36)$.

\subsubsection{ERP results}

As described in the Introduction, we hypothesized that participants would focus on retrieving specific episodic details for items studied recently but would be limited to retrieving relatively general information about items studied remotely. Our ERP analyses thus focused first on identifying retrieval orientation differences between the conditions compatible with these hypotheses: Recent-Specific and Remote-General. Similar to Experiment 1, this analysis employed a montage and latency periods determined by prior studies of retrieval orientation (e.g., Dzulkifli \& Wilding, 2005; Johnson \& McGhee, 2015; Johnson \& Rugg, 2006a, 2006b; Roberts et al., 2014). To anticipate the results, we observed no reliable effects across this montage, which did not warrant further tests (and permutation analysis) of the precise time course (cf. Experiment 1). Nevertheless, to investigate whether the retrieval orientation effects might be present on other electrodes, we next carried out permutation analyses of the topographic scalp maps. Finally, as the 
induction procedure developed and implemented here is novel, we further examined the effects of this procedure on the ERP data.

The ERPs associated with correctly rejected new items from the Recent-Specific and Remote-General test conditions are shown in Figure 5. As in Experiment 1, the ERPs for the recent test condition appear more positive-going than those for the remote condition, beginning by about $500 \mathrm{~ms}$ after stimulus onset. In contrast to the results of Experiment 1, however, the differences in the current experiment appear to be shorterlived and maximal over superior and midline electrodes, rather than being sustained and having a posterior distribution. To test for any retrieval orientation effect, the mean voltage amplitudes were extracted from each of the 15 electrodes shown in Figure 1 and from each of the predetermined latency periods $(200-500,500-800,800-1100,1100-$ 1400, 1400-1700, and 1700-2000 ms). An ANOVA of these data employed factors of orienting condition (Recent-Specific, Remote-General), anterior/posterior chain (frontal, central, and parietal), laterality (inferior left, superior left, midline, superior right, and inferior right), and latency period. We were interested only in the effects that included the orienting condition factor. Unlike Experiment 1, neither the omnibus ANOVA nor the subsidiary ANOVAs for each latency period yielded any significant effects.

To test whether the retrieval orientation effects in the current experiment might be evident outside the 15-electrode montage employed above, we also analyzed the full topographic maps of the Recent-Specific minus Remote-General differences. Those maps are provided in Figure 6. As shown, although there appears to be an anteriorly-distributed effect in the expected direction (more positive-going for Recent-Specific) from about 500 
to $1100 \mathrm{~ms}$ post-stimulus onset, this effect failed to meet the cluster-corrected level of significance in any of the latency periods.

Next, we investigated the effects of the induction manipulation that preceded each memory test block. Following the work of Madore and colleagues (e.g., Madore et al., 2014; Madore \& Schacter, 2016), we expected the effect of the induction phase to take the form of enhanced behavioral performance following the specific version; moreover, as we have generally found that the retrieval of recent items is associated with the highest levels of performance, we did not anticipate that the induction manipulation would influence recent-item retrieval to a large degree. (We return to the unexpected findings regarding this issue in the analyses below.) Based on these assumptions, we focused the analysis of the induction effect for new-item ERPs from the Remote-Specific and Remote-General tests. The ERPs associated with these two conditions, which are depicted in Figure 7, appear largely indistinguishable. To test for any effects, an ANOVA employing the 15-electrode montage and the latency periods from the retrieval orientation analyses was conducted on the mean voltage amplitudes. Whereas the omnibus ANOVA yielded a significant three-way interaction of induction condition, latency period, and anterior/posterior chain $(F(4.1,70.0)=2.89, p<.05)$, none of the subsidiary ANOVAs within each period yielded significant effects. We further attempted to address the nature of the three-way interaction with ANOVAs at each anterior/posterior chain, but again there were no significant effects. The effects that even approach significance (i.e., $p<.1$ ) are the induction condition $\times$ anterior/posterior chain interactions for the $1100-1400(F(1.4,23.3)=3.03, p=.084)$ and $1700-2000 \mathrm{~ms}(F(1.4$, 23.9) $=3.07, p=.081$ ) latency periods. Whereas the frontal ERPs are slightly more 
positive-going for the Remote-General condition in the earlier of these periods, in the latter period, the frontal effect is reversed and there is an accompanying positivity for Remote-General on the parietal chain.

As a final test of the effects of the induction manipulation, we focused on the Recent-General condition due to the fact that it gave rise to an unexpected pattern of findings. First, as shown in Figure 8, the ERPs for new items in this condition appeared more positive than the corresponding ERPs in the other three conditions, particularly from about 500 to $1000 \mathrm{~ms}$ post-stimulus onset. Second, as described above, although we anticipated that behavioral performance on the recent tests would be relatively unaffected by the induction procedure, performance was considerably lower in this compared to the other three test conditions (see Table 3). To further investigate the reliability of the ERP difference, we conducted an additional analysis comparing the putative remote vs. recent retrieval orientation effect following the general induction phase. With the 15 -electrode montage and latency periods used earlier, the mean ERP amplitudes for new items from the Remote-General and Recent-General conditions were submitted to a series of ANOVAs. The omnibus ANOVA revealed a significant two-way interaction of orienting condition $\times$ latency period $(F(2.6,43.8)=3.75, p<.05)$, as well as a significant threeway interaction of orienting condition $\times$ latency period $\times$ laterality $(F(4.4,75.3)=3.20, p$ $<.05)$. Follow-up ANOVAs within each latency period gave rise to significant main effects of orienting condition within the 200-500 $(F(1,17)=6.21, p<.05), 500-800$ $(F(1,17)=11.16, p<.01)$, and 800-1100 ms $(F(1,17)=5.93, p<.05)$ periods. Additionally, the condition $\times$ laterality interaction was significant during the $500-800 \mathrm{~ms}$ period $(F(2.2,26.8)=3.34, p<.05)$, reflecting the fact that the retrieval orientation effect 
appeared larger at superior and midline electrodes compared to inferior electrodes.

Follow-up $t$-tests at each level of the laterality factor confirmed that the 500-800 ms retrieval orientation effect was significant at each level (minimum $t(17)=2.78, p<.05$ )

Finally, permutation analyses of the topographic scalp maps of these effects, as shown in Figure 9, indicated significant clusters of electrodes in both the 500-800 and 800-1100 ms latency periods.

\subsection{SUMMARY}

The present experiment sought to replicate the effects in Experiment 1 (also see Johnson \& McGhee, 2015) of orienting retrieval to memories of different ages, and to additionally investigate the potential nature of the processing differences that underlie these effects. As described earlier, our hypothesis was that participants naturally focus on retrieving specific details to target items encoded recently, whereas they rely on more general episodic information to retrieve remote items. To this end, we developed a procedure analogous to one recently employed in multiple studies (e.g., Madore et al., 2015; Madore et al., 2014; Madore \& Schacter, 2014; Madore et al., 2016) in which we attempted to induce cognitive states of general or specific processing immediately prior to the start of each memory test.

In comparing the new-item ERPs for the remote and recent conditions that corresponded to our hypothesized forms of processing-Recent-Specific versus RemoteGeneral—we observed differences in the same direction (i.e., more positive for recent items) as those observed previously. However, these effects differed in that they did not reach significance, either with our standard 15-electrode montage or with topographic analyses, and they appeared to have a more-anterior distribution that before. We return to 
potential explanations of these discrepancies between experiments in the General Discussion.

Aside from the analyses of orienting effects for memory age, we also investigated other effects of the induction manipulation. Whereas the induction did not appear to affect the retrieval of items from the remote study list, in terms of both behavioral performance and the ERPs, it did give rise to unanticipated results on the recent test condition. In particular, the general induction led to significantly lower behavioral performance and more-positive going new-item ERPs for the following recent tests. Given the robustness of these effects-particularly in the face of the weak orienting effects we observed - we speculate about their meaning in the General Discussion. 


\section{Table 3}

Mean proportions of correct responses and corresponding RTs during the targeted memory tests in Experiment 2

\begin{tabular}{|l|c|c|c|c|}
\hline & \multicolumn{4}{|c|}{ Study condition } \\
\hline Targeted Week & \multicolumn{2}{|c|}{ Remote } & \multicolumn{2}{c|}{ Recent } \\
\hline Induction Type & General & Specific & General & Specific \\
\hline Proportions & $.86(.03)$ & $.85(.03)$ & $.83(.03)$ & $.91(.01)$ \\
\hline Target & $.91(.01)$ & $.91(.01)$ & $.85(.02)$ & $.90(.02)$ \\
\hline Nontarget & $.94(.01)$ & $.92(.02)$ & $.81(.02)$ & $.97(.01)$ \\
\hline New & $1231(43)$ & $1248(38)$ & $1178(35)$ & $1135(37)$ \\
\hline$R T s$ & $1200(33)$ & $1219(34)$ & $1303(47)$ & $1268(51)$ \\
\hline Target & $1231(51)$ & $1231(46)$ & $1207(42)$ & $1193(49)$ \\
\hline Nontarget
\end{tabular}

Note: SEMs are in parentheses. RTs are in milliseconds. 

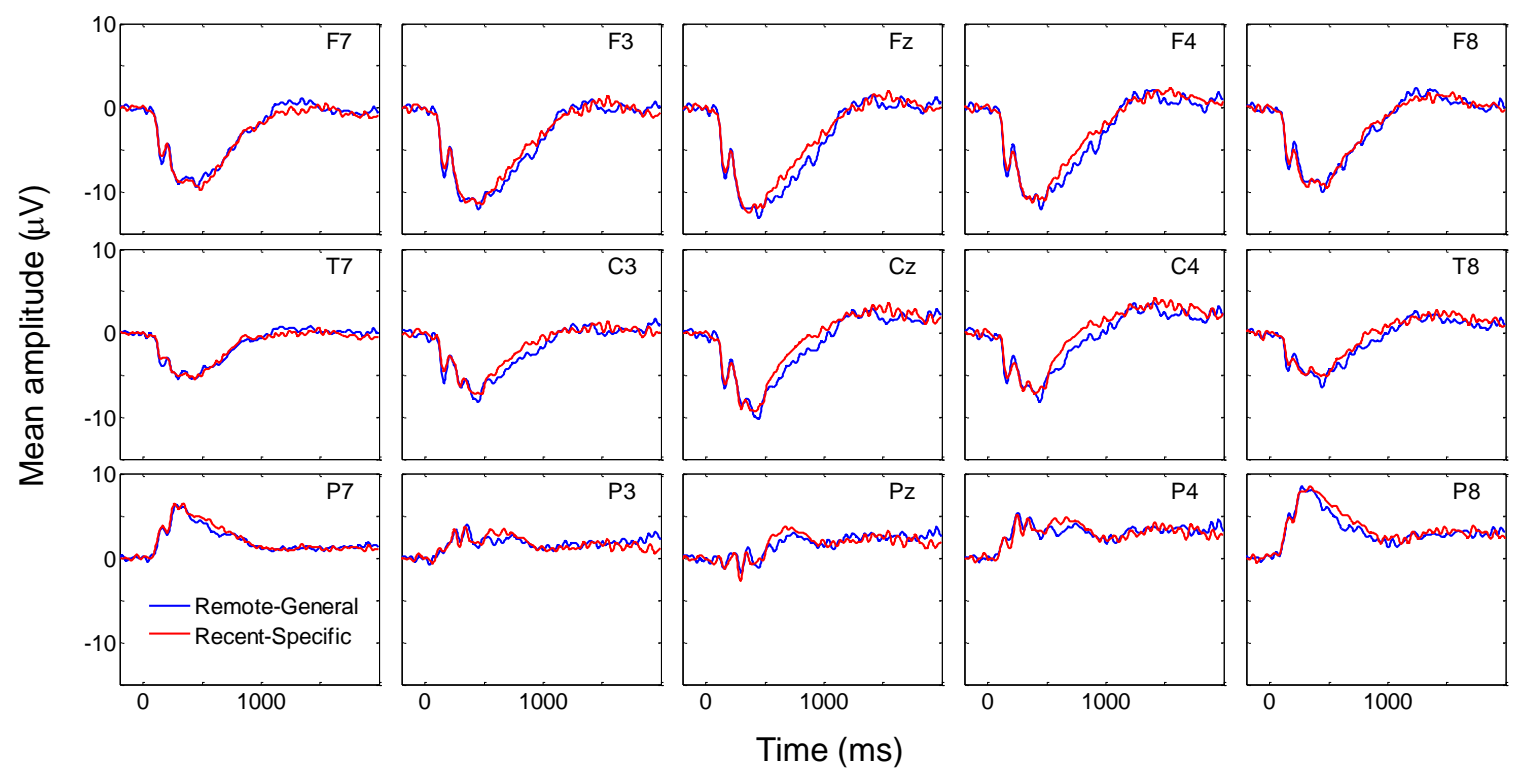

Figure 5. Mean ERPs during Remote-General and Recent-Specific orienting in

Experiment 2. Grand-average ERPs elicited by correctly rejected new items, according to whether Remote-General (blue) or Recent-Specific (red) memories were targeted. The electrodes corresponding to the ERPs are indicated in the upper right corner of each plot. 

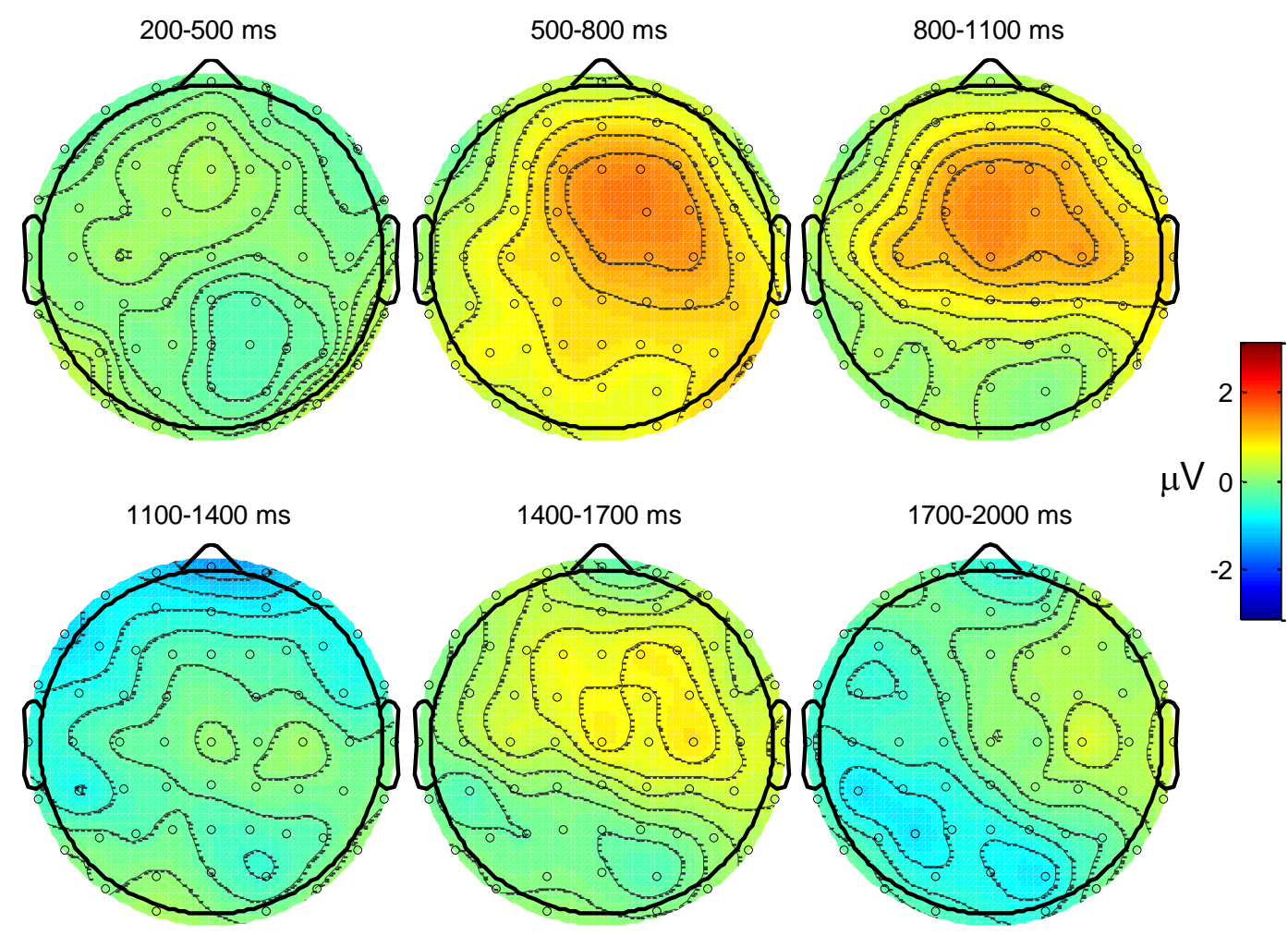

Figure 6. Topographic maps for the Recent-Specific minus Remote-General contrast in Experiment 2. Topographic maps resulting from the contrast of the RecentSpecific minus the Remote-General orienting condition within each predetermined latency period. 

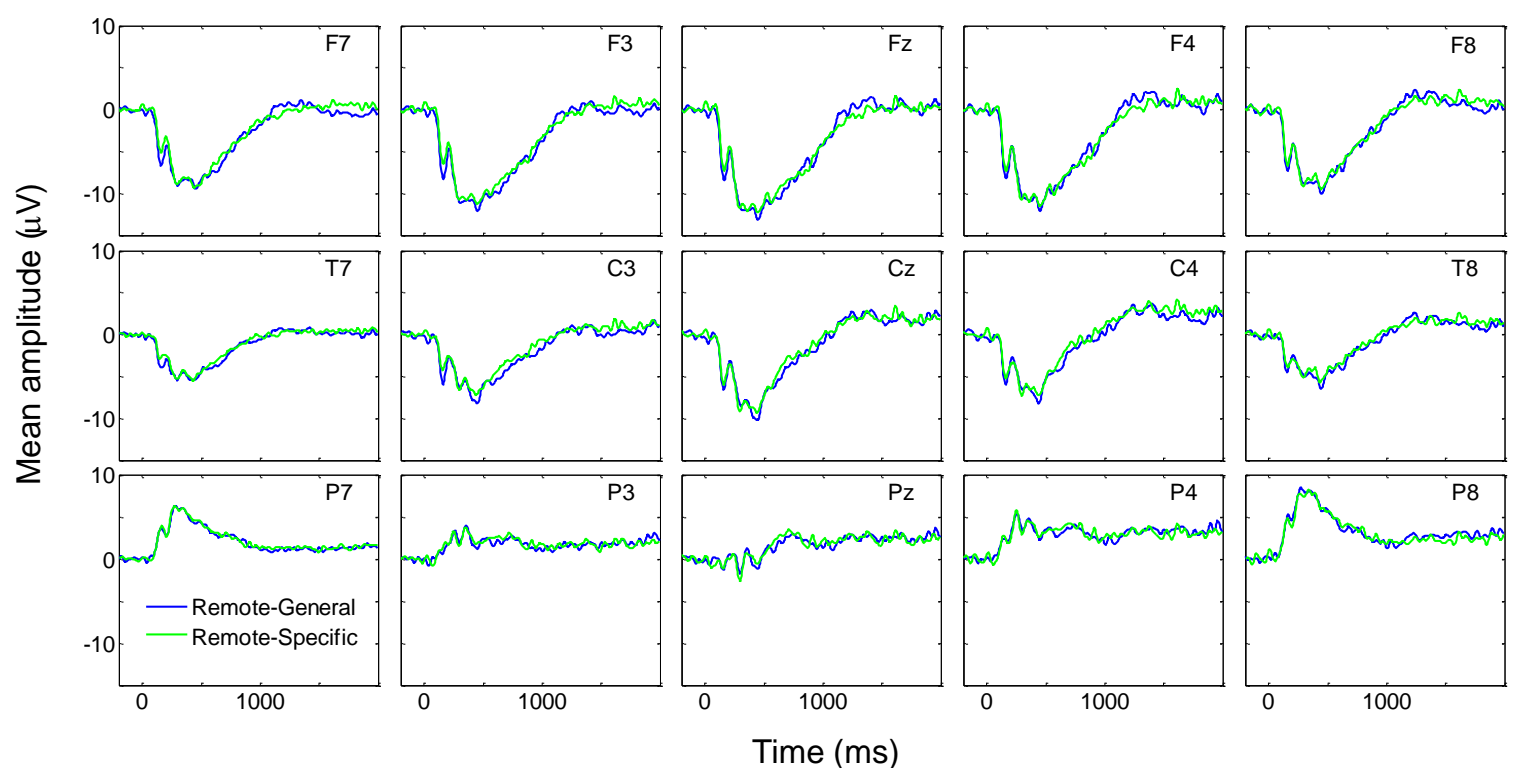

Figure 7. Mean ERPs during Remote-General and Remote-Specific orienting in

Experiment 2. Grand-average ERPs elicited by correctly rejected new items, according to whether Remote-General (blue) or Remote-Specific (green) memories were targeted. The electrodes corresponding to the ERPs are indicated in the upper right corner of each plot. 


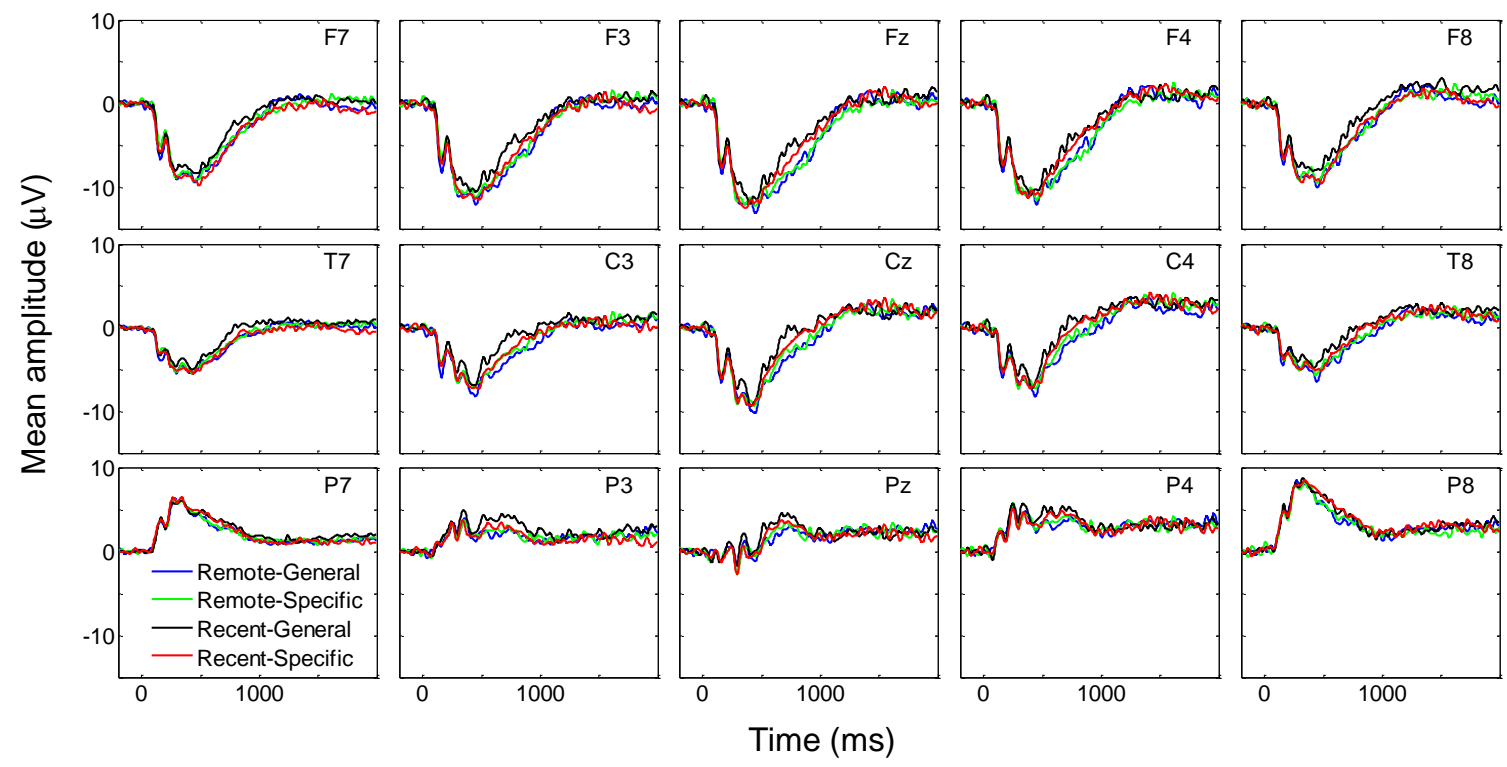

Figure 8. Mean ERPs during all four orienting conditions in Experiment 2.

Grand-average ERPs elicited by correctly rejected new items from Experiment 2, according to whether Remote-General (blue), Remote-Specific (green), Recent-General (black), or Recent-Specific (red) memories were targeted. The electrodes corresponding to the ERPs are indicated in the upper right corner of each plot. 

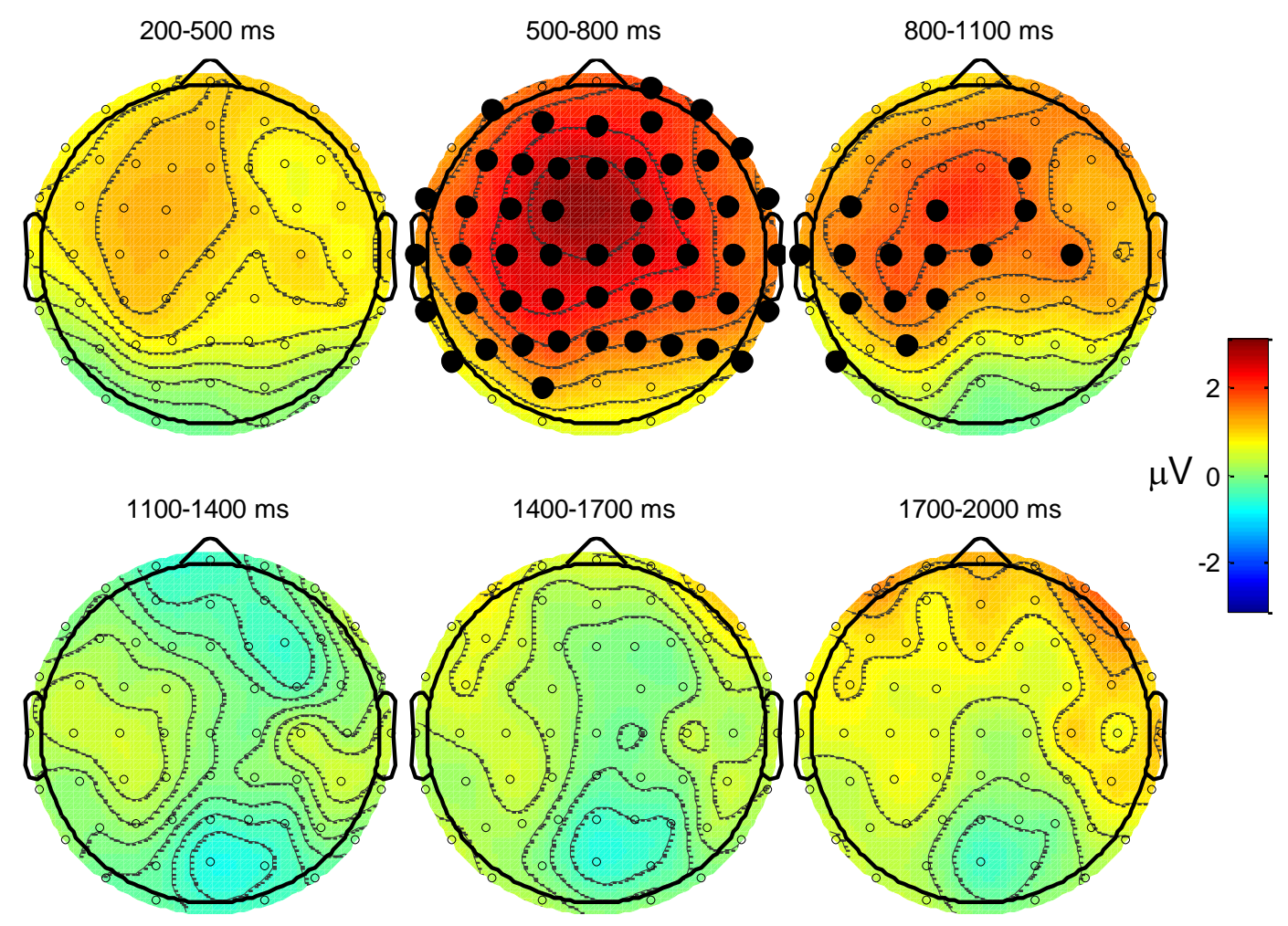

Figure 9. Topographic maps for the Recent-General minus Remote-General in

Experiment 2. Topographic maps resulting from the contrast of the Recent-General minus the Remote-General orienting condition within each predetermined latency period. Electrodes comprising significant clusters associated with the general induction effect are indicated with larger, filled circles. 


\section{Chapter 4}

\section{GENERAL DISCUSSION}

The experiments reported here investigated the differences in processing engaged when remote versus recent memories - i.e., those encoded one week versus about 30 minutes earlier - are to be retrieved. In Experiment 1, we examined whether the effects of orienting retrieval to memory age were dissociable from effects related to the difficulty of targeting remote memories. Of primary interest here was the comparison of strategic orienting to target memories for remote items that had been studied repeatedly (four times) and recent items that had been studied only once. We reasoned that increasing the number of study repetitions for remote items would strengthen the associated memory trace, bringing behavioral performance for this condition near the level of that for recent items studied once. In terms of both accuracy and the corresponding RTs associated with new test items, the repetition manipulation was successful in equating performance. Consistent with the effects of orienting retrieval to memory age that we observed previously, contrasting the ERPs elicited by correctly rejected new items during these equated conditions identified more-positive waveforms when orienting to recent memories (Johnson \& McGhee, 2015). As before, these effects again were evident by about $500 \mathrm{~ms}$ post-stimulus and were maximal over the posterior scalp. The findings of Experiment 1 thus extend the prior work by demonstrating that similar orienting differences occur when retrieval difficulty is controlled in an a priori manner. Moreover, 
Experiment 1 provides evidence that materials other than words, such as the pictures of objects employed here, can elicit such effects.

The repetition manipulation employed in Experiment 1 also allowed for the successful dissociation of differences putatively due to retrieval difficulty from those associated with orienting toward memory age. This dissociation was apparent in both the timing and the sign of the ERP effects. Whereas the repetition effect (as determined by the contrast of Remote-4x and Remote-1x) onset by around $200 \mathrm{~ms}$ and was short-lived, the effect for memory age (comparing Recent-1x and Remote-4x) did not onset until 500 ms and was sustained for about a full second. Further, the repetition effect took the form of positive-going waveforms for the more difficult, Remote-1x condition; whereas the opposite pattern emerged when identifying the orienting effect, with the easier condition of retrieving recent items (Recent-1x) eliciting positive-going waveforms (also see Johnson \& McGhee, 2015). These findings together support the idea that, when participants attempt to retrieve remote versus recent memories, they are engaging processes at least partly distinct from those engaged to overcome retrieval difficulty.

Although it was not relevant to the main analyses of the ERPs to identify orienting effects, another noteworthy aspect of the results of Experiment 1 is the lack of behavioral differences in the final test phase for items presented earlier in the context of remote and recent orienting. These findings are in contrast to several recent papers that employed a similar test phase (e.g., Danckert, MacLeod, \& Fernandes, 2011; Jacoby et al., 2005; Marsh et al., 2009; Shimizu \& Jacoby, 2005; Vogelsang, Bonnici, Bergström, Ranganath, \& Simons, 2016). For example, in Vogelsang et al. (2016), items that were presented as new items during the context of retrieving deeply encoded memories were 
more likely to be retrieved on a subsequent test than items presented in the context of retrieving shallowly encoded memories. The discrepant findings between those studies and Experiment 1 could be due to several factors. First, it is possible that the levels-ofprocessing (deep vs. shallow) manipulation of those studies is more powerful than the remote-recent manipulation we employed. Second, the pictures used in the present study gave rise to better memory performance on the final test, compared to the words employed by Vogelsang et al. The absence of a difference according to previous orienting condition in Experiment 1 could thus be due to the restricted range available for observing an effect. This particular explanation cannot account for the previous failure to observe such effects with word stimuli (Johnson \& McGhee, 2015), but as described in that paper, several participants reported being confused about the task for the final test phase. Further studies directed at bringing together these different types of manipulations in terms of behavioral consequences on memory are clearly needed.

In Experiment 2, we tested the hypothesis that orienting to remote or recent memories was associated with differences in the level of specificity of episodic retrieval processing. A novel procedure was developed and implemented to induce general or specific states of cognitive processing just prior to completing each memory test block. Whereas previous work has examined the influence of specificity via a Cognitive Interview paradigm (Madore et al., 2015; Madore et al., 2014; Madore \& Schacter, 2014; Madore et al., 2016), our study is the first to our knowledge to do so with a computerbased procedure that manipulated the degree of induced specificity. The potential usefulness of such a procedure lies in allowing for the testing of different retrieval conditions (e.g., our remote and recent tests) that shortly follow the induction phases, 
whereas the lengthy and short-lived nature of the Cognitive Interview, by contrast, appears to limit such flexibility and repeated use. Using this novel induction procedure, our main hypothesis was that remote memories should be associated with attempts to recover fewer details than recent memories, and that strategic orienting to remote and recent memories would therefore focus on lesser and greater degrees of specificity, respectively. We thus initially compared the ERPs for recent orienting following a specific induction and remote orienting following a general induction. Contrary to our hypothesis and previous results, although the ERPs for these two conditions in Experiment 2 appeared to diverge in about the same time period and were in the same direction as those demonstrated previously, they failed to reach significance.

One possible explanation for the aforementioned absence of orienting effects in Experiment 2 is related to our use of relatively short blocks in the targeted test phase. We adopted this design due to the potential concern that the effects of the episodic specificity induction would be relatively short-lived (also see Madore \& Schacter, 2014). However, it is possible that shortening the tests had the unintended consequence of limiting the amount of time that participants were able to "settle into" a particular cognitive state. Indeed, we and others have previously observed that multiple trials are required for the ERPs from different orienting conditions to begin to diverge (Johnson \& Rugg, 2006a). Moreover, it is possible that even longer sequences of trials are necessary to begin to observe statistically reliable orienting differences. Unfortunately, testing the comparable orienting effect based on the initial 26 trials (i.e. the length of test blocks in Experiment 2) in Experiment 1 is not anticipated to be reliable given that there was only one block per condition in that experiment (compared to 3 blocks in Experiment 2). 
An alternative explanation for the weak effects of orienting to memory age in Experiment 2 is that only including the condition of repeated items during the remote study list caused subjects to not orient retrieval processes to the same degree as in the previous experiment. By this account, subjects might be more likely to engage in a particular strategy when retrieval is perceived as somewhat difficult. This perception of difficulty could have been highlighted by the remote study condition in which items were presented only once, as in Experiment 1 and in Johnson and McGhee (2015), but may have been absent in the current experiment. The lack of behavioral and ERP effects associated with the different induction conditions when preceding the remote test is compatible with this latter account. In addition, previous work demonstrating the mnemonic benefit of an episodic specificity induction has only tested memories created in the laboratory over material studied that same day, usually within a period of two to three hours (Madore \& Schacter, 2016). Experiment 2 is the first study, to our knowledge, to investigate the impact of an episodic specificity induction on remote memories encoded in the laboratory (but see Madore et al., 2014, for a similar approach with remote autobiographical memories). Future studies can begin to address these ideas by expanding the use of induction procedures beyond the realm of recently encoded memories formed in the laboratory (e.g., beyond the 2-3 hour retention intervals used by Madore \& Schacter, 2016).

Despite the lack of significant retrieval orientation effects in Experiment 2, the effect of the induction manipulation on recent memory retrieval is consistent with previous work (Madore \& Schacter, 2016). Specifically, the behavioral performance on recent items following the specific induction was better than that following the general 
induction. Despite this consistency, the Recent-General condition was associated with reduced memory performance and more positive ERPs, relative to the other three conditions. The fact that these effects seem to stand out for the Recent-General condition raises the question of whether the induction effects might have more to do with the general induction than the specific induction. That is, does the specific induction help memory, as we originally interpreted the prior findings (e.g., Madore \& Schacter, 2016), or does the general induction instead lead to a memory deficit? This issue could be addressed in follow-up studies by including a baseline condition-perhaps consisting of no induction phase - against which the two inductions can be tested.

Although Experiment 2 did not provide strong evidence in support of our hypothesized retrieval orientation effects, the partial support we did obtain for the effectiveness of the induction on orienting to recent memories is encouraging in terms of the potential utility of related procedures for modulating memory. More work is clearly needed to evaluate the effectiveness of our particular procedure, especially outside the context of studies where retrieval orientation is the main focus. Ideally, such procedures could have important implications for elderly or clinical populations. Older adults tend to generate fewer and less-specific details than young adults for both past memories and imagined future events (Addis, Musicaro, Pan, \& Schacter, 2010), and there is evidence that inducing specific episodic processing leads to a brief improvement in these abilities (Madore et al., 2014). Furthermore, a number of different clinical populations have been shown to exhibit a common pattern of retrieving overgeneralized autobiographical memories, including individuals with depression, schizophrenia, and post-traumatic stress disorder (Brown et al., 2014; Brown et al., 2013; D’Argembeau, Raffard, \& Van der 
Linden, 2008; Hach, Tippett, \& Addis, 2014; Williams et al, 1996). Some recent studies have demonstrated that extensive training on retrieving specific memories may mitigate this overgeneralization in individuals with depression (Neshat-Doost et al., 2013; Reas, William, \& Hermans, 2009). Thus, further investigation of the effectiveness of our induction method and related training methods could have significant translational value. 


\section{REFERENCES}

Addis, D. R., Musicaro, R., Pan, L., \& Schacter, D. L. (2010). Episodic simulation of past and future events in older adults: Evidence from an experimental recombination task. Psychology and Aging, 25(2), 369-376. https://doi.org/10.1037/a0017280

Allan, K., Wilding, E. L., \& Rugg, M. D. (1998). Electrophysiological evidence for dissociable processes contributing to recollection. Acta Psychologia, 98(2-3), 231-252. https://doi.org/10.1016/S0001-6918(97)00044-9

Balota, D. A., Dolan, P. O., \& Duchek, J. M. (2000). Memory changes in healthy young and older adults. In E. Tulving \& F. I. M. Craik (Eds.), The Oxford Handbook of Memory (pp. 395-410). Oxford: Oxford University Press.

Brown, A. D., Addis, D. R., Romano, T. A., Marmar, C. R., Bryant, R. A., Hirst, W., \& Schacter, D. L. (2014). Episodic and semantic components of autobiographical memories and imagined future events in post-traumatic stress disorder. Memory, 22(6), 595-604. https://dx.doi.org/10.1080/09658211.2013.807842

Brown, A. D., Root, J. C., Romano, T. A., Chang, L. J., Bryant, R. A., \& Hirst, W. (2013). Overgeneralized autobiographical memory and future thinking in combat veterans with posttraumatic stress disorder. Journal of Behavior Therapy and Experimental Psychiatry, 44(1), 129-134. https://doi.org/10.1016/j.jbtep.2011.11.004

Buckner, R. L. (2003). Functional-anatomic correlates of control processes in memory. Journal of Neuroscience, 23(10), 3999-4004. 
Buckner, R. L. (2004). Memory and executive function in aging and AD: Multiple factors that cause decline and reserve factors that compensate. Neuron, 44(1), 195-208. https://doi.org/10.1016/j.neuron.2004.09.006

Buckner, R. L., Koutstaal, W., Schacter, D. L., Wagner, A. D., \& Rosen, B. R. (1998). Functional-anatomic study of episodic retrieval using fMRI: Retrieval effort versus retrieval success. NeuroImage, 7, 151-162. https://doi.org/10.1006/nimg.1998.0327

Burke, D. M., \& Light, L. L. (1981). Memory and aging: The role of retrieval processes. Psychological Bulletin, 90(3), 513-546. https://doi.org/10.1037/00332909.90.3.513

Chaumon, M., Bishop, D. V., \& Busch, N. A. (2015). A practical guide to the selection of independent components of the electroencephalogram for artifact correction. Journal of Neuroscience Methods, 250, 57-63. https://doi.org/10.1016/j.jneumeth.2015.02.025

D’Argembeau, A., Raffard, S., \& Van der Linden, M. (2008). Remembering the past and imagining the future in schizophrenia. Journal of Abnormal Psychology, 117(1), 247-251. https://doi.org/10.1037/0021-843X.117.1.247

Danckert, S. L., MacLeod, C. M., \& Fernandes, M. A. (2011). Source-constrained retrieval influences the encoding of new information. Memory and Cognition, 39(8), 1374-1386. https://doi.org/10.3758/s13421-011-0117-9 
Davis, H. P., Small, S. A., Stern, Y., Mayeux, R., Feldstein, S. N., \& Keller, F. R. (2003). Acquisition, recall, and forgetting of verbal information in long-term memory by young, middle-aged, and elderly individuals. Cortex, 39, 1063-1091. https://doi.org/10.1016/S0010-9452(08)70878-5

Delorme, A., \& Makeig, S. (2004). EEGLAB: An open source toolbox for analysis of single-trial EEG dynamics including independent component analysis. Journal of Neuroscience Methods, 134, 9-21. https://doi.org/10.1016/j.jneumeth.2003.10.009

Dewar, M. T., Cowan, N., \& Della Sala, S. (2007). Forgetting due to retroactive interference: A fusion of Müller and Pilzecker's (1900) early insights into everyday forgetting and recent research on anterograde amnesia. Cortex, 43, 616634. https://doi.org/10.1016/S0010-9452(08)70492-1

Donaldson, D. I., Allan, K. \& Wilding, E. L. (2002). Fractionating episodic memory retrieval using event-related potentials. In A. Parker, E. L. Wilding, \& T. J. Bussey (Eds.), The Cognitive Neuroscience of Memory: Encoding and Retrieval (pp. 39-58). London: Psychology Press.

Dudai, Y. (2004). The neurobiology of consolidations, or, how stable is the engram? Annual Review of Psychology, 55, 51-86. https://doi.org/10.1146/annurev.psych.55.090902.142050

Dzulkifli, M. A., Sharpe, H. L., \& Wilding, E. L. (2004). Separating item-related electrophysiological indices of retrieval effort and retrieval orientation. Brain and Cognition, 55, 433-443. https://doi.org/10.1016/j.bandc.2004.03.004 
Dzulkifli, M. A., \& Wilding, E. L. (2005). Electrophysiological indices of strategic episodic retrieval processing. Neuropsychologia, 43(8), 1152-1162. https://doi.org/10.1016/j.neuropsychologia.2004.11.019

Fisher, R. P., \& Geiselman, R. E. (1992). Memory enhancing techniques for investigative interviewing: The cognitive interview. Springfield, IL: Charles C. Thomas.

Frankland, P. W., \& Bontempi, B. (2005). The organization of recent and remote memories. Nature Reviews Neuroscience, 6, 119-130. https://doi.org/10.1038/nrn1607

Friedman, D. (2000). Event-related brain potential investigations of memory and aging. Biological Psychiatry, 54(1-3), 175-206. https://doi.org/10.1080/09658210143000353

Friedman, D., de Chastelaine, M., Nessler, D., \& Malcolm, B. (2010). Changes in familiarity and recollection across the lifespan: An ERP perspective. Brain Research, 1310, 124-141. https://doi.org/10.1016/j.brainres.2009.11.016

Gardner, J. M., \& Java, R. I. (1991). Forgetting in recognition memory with and without recollective experience. Memory \& Cognition, 19(6), 617-623. https://doi.org/10.3758/BF03197157

Hach, S., Tippett, L. J., \& Addis, D. R. (2014). Neural changes associated with the generation of specific past and future events in depression. Neuropsychologia, 65, 41-55. https://doi.org/10.1016/j.neuropsychologia.2014.10.003

Herron, J. E., Evans, L. H., \& Wilding, E. L. (2016). Electrophysiological evidence for flexible goal-directed cue processing during episodic retrieval. NeuroImage, 132, 24-31. https://doi.org/10.1016/j.neuroimage.2016.02.025 
Herron, J. E., \& Rugg, M. D. (2003a). Retrieval orientation and the control of recollection. Journal of Cognitive Neuroscience, 15(6), 843-854. https://doi.org/10.1162/089892903322370762

Herron, J.E., Rugg, M.D. (2003b). Strategic influences on recollection in the exclusion task: electrophysiological evidence. Psychonomic Bulletin \& Review, 10, 703710. https://doi.org/10.3758/BF03196535

Herron, J. E., \& Wilding, E. L. (2004). An electrophysiological dissociation of retrieval mode and retrieval orientation. NeuroImage, 22(4), 1554-1562. https://doi.org/10.1016/j.neuroimage.2004.04.011

Herron, J. E., \& Wilding, E. L. (2005). An electrophysiological investigation of factors facilitating strategic recollection. Journal of Cognitive Neuroscience, 17(5), 777787. https://doi.org/10.1162/0898929053747649

Hinrichs, J. V. (1970). A two-process memory-strength theory for judgment of recency. Psychological Review, 77(3), 223-233. doi: 10.1037/h0029101

Hintzman, D. L. (2002). Context matching and judgments of recency. Psychonomic Bulletin \& Review, 9(2), 368-374. https://doi.org/10.3758/BF03196295

Hintzman, D. L. (2005). Memory strength and recency judgments. Psychonomic Bulletin \& Review, 12(5), 858-864. https://doi.org/10.3758/BF03196777

Hintzman, D. L., Block, R. A., \& Summers, J. J. (1973). Contextual associations and memory for serial position. Journal of Experimental Psychology, 97(2), 220-229. https://doi.org/10.1037/h0033884 
Hornberger, M., Morcom, A. M., \& Rugg, M. D. (2004). Neural correlates of retrieval orientation: effects of study-test similarity. Journal of Cognitive Neuroscience, 16(7), 1196-1210. https://doi.org/10.1162/0898929041920450

Howard, M. W., \& Kahana, M. J. (2002). A distributed representation of temporal context. Journal of Mathematical Psychology, 46, 269-299. https://doi.org/10.1006/jmps.2001.1388

Jacoby, L. L. (1991). A process dissociation framework: Separating automatic from intentional uses of memory. Journal of Memory and Language, 30, 513-541. https://doi.org/10.1016/0749-596X(91)90025-F

Jacoby, L.L., 1998. Invariance in automatic influences of memory: toward a user's guide for the process-dissociation procedure. Journal of Experimental Psychology: Learning, Memory, and Cognition, 24, 3-26. https://doi.org/10.1037/02787393.24.1.3

Jacoby, L. L., Kelley, C. M., \& McElree, B. D. (1999). The role of cognitive control: Early selection versus late correction. In S. Chaiken \& Y. Trope (Eds.), DualProcess Theories in Social Psychology (pp. 383-400). New York: The Guildford Press.

Jacoby, L. L., Shimizu, Y., Daniels, K. A., \& Rhodes, M. G. (2005). Modes of cognitive control in recognition and source memory: Depth of retrieval. Psychonomic Bulletin \& Review, 12, 852-857. https://doi.org/10.3758/BF03196776 
Jacoby, L.L., Toth, J. P., \& Yonelinas, A. P. (1993). Separating conscious and unconscious influences of memory: Measuring recollection. Journal of Experimental Psychology: General, 122(2), 139-154. https://doi.org/10.1037/0096-3445.122.2.139

Johnson, J. D., \& McGhee, A. K. (2015). Electrophysiological evidence for strategically orienting retrieval toward the specific age of a memory. Brain and Cognition, 100, 41-48. https://doi.org/10.1016/j.bandc.2015.09.007

Johnson, J. D., \& Rugg, M. D. (2006). Modulation of the electrophysiological correlates of retrieval cue processing by the specificity of task demands. Brain Research, 1071, 153-164. https://doi.org/10.1016/j.brainres.2005.11.093

Johnson, M. K., Kounios, J., \& Nolde, S. F. (1997). Electrophysiological brain activity and memory source monitoring. Neuroreport, 8(5), 1317-1320. https://doi.org/10.1097/00001756-199703240-00051

Levine, B., Svoboda, E., Hay, J. F., Winocur, G., \& Moscovitch, M. (2002). Aging and autobiographical memory: Dissociating episodic from semantic retrieval. Psychology and Aging, 17(4), 677-689. https://doi.org/10.1037/08827974.17.4.677

Luo, L., \& Craik, F. I. (2008). Aging and memory: a cognitive approach. Canadian Journal of Psychiatry, 53(6), 346-353. https://doi.org/10.1177/070674370805300603

Madore, K. P., Addis, D. R., \& Schacter, D. L. (2015). Creativity and memory: Effects of an episodic-specificity induction on divergent thinking. Psychological Science, 26(9), 1461-1468. https://doi.org/10.1177/0956797615591863 
Madore, K. P., Gaesser, B., \& Schacter, D. L. (2014). Constructive episodic simulation: Dissociable effects of a specificity induction on remembering, imagining, and describing in young and older adults. Journal of Experimental Psychology:

Learning, Memory, and Cognition, 40(3), 609-622.

https://doi.org/10.1037/a0034885

Madore, K. P., \& Schacter, D. L. (2014). An episodic specificity induction enhances means-end problem solving in young and older adults. Psychology and Aging, 29(4), 913-924. https://doi.org/10.1037/a0038209

Madore, K. P., \& Schacter, D. L. (2016). Remembering the past and imagining the future: Selective effects of an episodic specificity induction on detail generation. The Quarterly Journal of Experimental Psychology, 69, 285-298. https://doi.org/10.1080/17470218.2014.999097

Madore, K. P., Szpunar, K. K., Addis, D. R., \& Schacter, D. L. (2016). Episodic specificity induction impacts activity in a core brain network during construction of imagined future experiences. Proceedings of the National Academy of Sciences of the United States of America, 113(38), 10696-10701. https://doi.org/10.1073/pnas.1612278113

Maris, E., \& Oostenveld, R. (2007). Nonparametric statistical testing of EEG- and MEGdata. Journal of Neuroscience Methods, 164, 177-190.

https://doi.org/10.1016/j.jneumeth.2007.03.024 
Marsh, R. L., Meeks, J. T., Cook, G. I., Clark-Foos, A., Hicks, J. L., \& Brewer, G. A. (2009). Retrieval constraints on the front end create differences in recollection on a subsequent test. Journal of Memory and Language, 61, 470-479. https://doi.org/10.1016/j.jml.2009.06.005

McGaugh, J. L. (2000). Memory--a century of consolidation. Science, 287(5451), 248251. https://doi.org/10.1126/science.287.5451.248

Mitchell, D. B., Brown, A. S., \& Murphy, D. R. (1990). Dissociations between procedural and episodic memory: Effects of time and aging. Psychology and Aging, 5(2), 264-276. https://doi.org/10.1037/0882-7974.5.2.264

Morcom, A. M. (2016). Mind over memory: Cuing the aging brain. Current Directions in Psychological Science, 25(3), 143-150. https://doi.org/10.1177/0963721416645536

Morcom, A. M., \& Rugg, M. D. (2004). Effects of age on retrieval cue processing as revealed by ERPs. Neuropsychologia, 42(11), 1525-1542. https://doi.org/10.1016/j.neuropsychologia.2004.03.009

Morris, C. D., Bransford, J. D., \& Franks, J. J. (1977). Levels of processing versus transfer appropriate processing. Journal of Verbal Learning and Verbal Behavior, 16, 519-533. https://doi.org/10.1016/S0022-5371(77)80016-9

Moscovitch, M., Cabeza, R., Winocur, G., \& Nadel, L. (2016). Episodic memory and beyond: The hippocampus and neocortex in transformation. Annual Review of Psychology, 67, 105-134. https://doi.org/10.1146/annurev-psych-113011-143733 
Nadel, L., Campbell, J., \& Ryan, L. (2007). Autobiographical memory retrieval and hippocampal activation as a function of repetition and the passage of time. Neural Plasticity, 2007, 90472. https://doi.org/10.1155/2007/90472

Neshat-Doost, H. T., Dalgleish, T., Yule, W., Kalantari, M., Ahmadi, S. J., Dyregrov, A., \& Jobson, L. (2013). Enhancing autobiographical memory specificity through cognitive training: An intervention for depression translated from basic science. Clinical Psychological Science, 1(1), 84-92. https://dx.doi.org/10.1177\%2F2167702612454613

Piefke, M., Weiss, P. H., Zilles, K., Markowitsch, H. J., \& Fink, G. R. (2003). Differential remoteness and emotional tone modulate the neural correlates of autobiographical memory. Brain, 126(3), 650-668. https://doi.org/10.1093/brain/awg064

Piolino, P., Desgranges, B., Benali, K. \& Eustache, F. (2002). Episodic and semantic autobiographical memory in ageing. Memory, 10, 239-257. https://doi.org/10.1080/09658210143000353

Reas, F., Williams, J. M. G., \& Hermans, D. (2009). Reducing cognitive vulnerability to depression: A preliminary investigation of MEmory Specificity Training (MEST) in inpatients with depressive symptomatology. Journal of Behavior Therapy and Experimental Psychiatry, 40(1), 24-38.

https://doi.org/10.1016/j.jbtep.2008.03.001

Robb, W. G. K., \& Rugg, M. D. (2002). Electrophysiological dissociation of retrieval orientation and retrieval effort. Psychonomic Bulletin \& Review, 9, 583-589. https://doi.org/10.3758/BF03196316 
Roberts, J. S., Tsivilis, D., \& Mayes, A. R. (2014). Strategic retrieval processing and the impact of knowing when a memory was first created. Brain and Cognition, 86, 124-130. https://doi.org/10.1016/j.bandc.2014.02.005

Roediger, H. L., III, Weinstein, Y., \& Agarwal, P. K. (2010). Forgetting: preliminary considerations. In S. Della Sala (Ed.), Forgetting (pp. 1-22). New York: Psychology Press.

Rosburg, T., Johansson, M., \& Mecklinger, A. (2013). Strategic retrieval and retrieval orientation in reality monitoring studied by event-related potentials.

Neuropsychologia, 51, 557-571.

https://doi.org/10.1016/j.neuropsychologia.2012.11.014

Rosburg, T., Johansson, M., Sprondel, V., \& Mecklinger, A. (2014). Retrieving selfvocalized information: An event-related potential (ERP) study on the effect of retrieval orientation. Brain \& Cognition, 92, 123-132. https://doi.org/10.1016/j.bandc.2014.10.011

Rudoy, J. D., Weintraub, S., \& Paller, K. A. (2009). Recall of remote episodic memories can appear deficient because of a gist-based retrieval orientation. Neuropsychologia, 47(3), 938-941. https://doi.org/10.1016/j.neuropsychologia.2008.12.006

Rugg, M. D., Allan, K., \& Birch, C. S. (2000). Electrophysiological evidence for the modulation of retrieval orientation by depth of study processing. Journal of Cognitive Neuroscience, 12, 664-678. https://doi.org/10.1162/089892900562291 
Rugg, M. D., Henson, R. N. A., \& Robb, W. G. K. (2003). Neural correlates of retrieval processing in the prefrontal cortex during recognition and exclusion tasks. Neuropsychologia, 41(1), 40-52. https://doi.org/10.1016/S0028-3932(02)00129-X

Rugg, M. D., Johnson, J. D., Park, H., \& Uncapher, M. R. (2008). Encoding-retrieval overlap in human episodic memory: a functional neuroimaging perspective. Progress in Brain Research, 169, 339-352. https://doi.org/10.1016/S00796123(07)00021-0

Rugg, M. D., \& Wilding, E. L. (2000). Retrieval processing and episodic memory. Trends in Cognitive Sciences, 4, 108-115. https://doi.org/10.1016/S13646613(00)01445-5

Schacter, D. L., \& Madore, K. P. (2016). Remembering the past and imagining the future: Identifying and enhancing the contribution of episodic memory. Memory Studies, 9(3), 245-255. https://doi.org/10.1177/1750698016645230

Schmolck, H., Buffalo, E. A., \& Squire, L. R. (2000). Memory distortions develop over time: Recollection of the O.J. Simpson trial verdict after 15 and 32 months. Psychological Science, 11(1), 39-45. https://doi.org/10.1111/1467-9280.00212

Shimizu, Y., \& Jacoby, L. L. (2005). Similarity-guided depth of retrieval: Constraining at the front end. Canadian Journal of Experimental Psychology, 59, 17-21. https://doi.org/10.1037/h0087455

Squire, L. R. (1989). On the course of forgetting in very long-term memory. Journal of Experimental Psychology: Learning, Memory, and Cognition, 15, 241-245. https://doi.org/10.1037/0278-7393.15.2.241 
Talamini, L. M., \& Gorree, E. (2012). Aging memories: Differential decay of episodic memory components. Learning \& Memory, 19, 239-246. https://doi.org/10.1101/lm.024281.111

Tulving, E. (1983). Elements of episodic memory. Oxford: Clarendon Press.

Tulving E., \& Osler, A. (1968). Effectiveness of retrieval cues in memory for words. Journal of Experimental Psychology, 77(4), 593-601. https://doi.org/10.1037/h0026069

Tulving, E., \& Thomson, D. M. (1973). Encoding specificity and retrieval processes in episodic memory. Psychological Review, 80, 352-373. https://doi.org/10.1037/h0020071

Vogelsang, D. A., Bonnici, H. M., Bergström, Z. M., Ranganath, C., \& Simons, J. S. (2016) Goal-directed mechanisms that constrain retrieval predict subsequent memory for new "foil” information. Neuropsychologia, 89, 356-363. https://doi.org/10.1016/j.neuropsychologia.2016.07.016

Wickelgren, W. A. (1974). Single-trace fragility theory of memory dynamics. Memory \& Cognition, 2, 775-780. https://doi.org/10.3758/BF03198154.

Wilckens, K. A., Erickson, K. I., \& Wheeler, M. E. (2012). Age-related decline in controlled retrieval: The role of the PFC and sleep. Neural Plasticity, 2012, 624795. https://doi.org/10.1155/2012/624795

Wilding, E. L. (1999). Separating retrieval strategies from retrieval success: an eventrelated potential study of source memory. Neuropsychologia, 37, 441-454. https://doi.org/10.1016/S0028-3932(98)00100-6 
Wilding, E. L., \& Nobre, A. C. (2001). Task-switching and memory retrieval processing: Electrophysiological evidence. NeuroReport, 12(16), 3613-3617. https://doi.org/10.1097/00001756-200111160-00048

Wilding, E. L., \& Rugg, M. D. (1997). Event-related potentials and the recognition memory exclusion task. Neuropsychologia, 35(2), 119-128. https://doi.org/10.1016/S0028-3932(96)00076-0

Williams, J. M. G., Ellis, N. C., Tyers, C., Healy, H., Rose, G., \& Macleod, A. K. (1996). The specificity of autobiographical memory and imageability of the future. Memory \& Cognition, 24(1), 116-125. https://doi.org/10.3758/BF03197278

Wixted, J. T. (2004). The psychology and neuroscience of forgetting. Annual Review of Psychology, 55, 235-269. https://doi.org/10.1146/annurev.psych.55.090902.141555

Wixted, J. T. (2005). A theory about why we forget what we once knew. Current Directions in Psychological Science, 14(1), 6-9. https://doi.org/10.1111/j.09637214.2005.00324.x 


\section{VITA}

Emily Leiker was born and raised in Kansas, the oldest of three children. She spent most of her childhood in Olathe, running around the neighborhood and exploring the nearby parks and trails. Emily showed an early interest in understanding the world around her, preferring to spend her time either outside studying frogs, slugs, ants, and other insects or wildlife, or inside reading books about them. Her love of science and learning eventually led her to attend Drake University in Des Moines, Iowa, where she developed a passion for understanding the connections between the brain, cognition, and behavior. She graduated magna cum laude and with University Honors from Drake in 2011, earning degrees in both Neuroscience and Psychology. During her time at Drake, Emily fueled her passion for neuroscience as a research assistant in the laboratory of Dr. Olga Lazareva, whose fierceness and confidence emboldened Emily to pursue graduate study in psychology and neuroscience, and join the ranks of women in academia.

Emily was awarded the prestigious Life Sciences Fellowship to pursue her doctorate in the Department of Psychological Sciences at the University of Missouri in 2011, where she completed her graduate studies in the Cognition and Neuroscience training area under the mentorship of Dr. Jeff Johnson. Through her work in Dr. Johnson's Memory \& Neuroimaging Lab, Emily developed the skills, confidence, and expertise needed to establish a successful independent career in cognitive neuroscience research. She will graduate in May 2017, after which she plans to complete several postdoctoral appointments to further her training in neuroimaging approaches for studying human memory, before ultimately establishing a research lab of her own. 